\title{
INVESTIGATION OF THE RH-CATALYZED ASYMMETRIC REDUCTIVE ALDOL REACTION. EXPANDED SCOPE BASED ON REACTION ANALYSIS.
}

\author{
Albert E. Russell, Nathan O. Fuller, Steven J. Taylor, Pauline Aurissett and \\ James P. Morken* \\ Department of Chemistry, Venable and Kenan Laboratories \\ University of North Carolina, Chapel Hill, NC 27599-3290
}

\section{Supplementary Material}

General. Infrared spectra were recorded on a Nicolet Magna 560 spectrometer, $v_{\max } \mathrm{in}_{\mathrm{cm}}^{-1}$. Bands are characterized as broad (br), strong (s), medium (m), and weak (w). ${ }^{1} \mathrm{H}$ NMR spectra were recorded on Varian Gemini (300 MHz) and Bruker (400 MHz) spectrometers. Chemical shifts are reported in ppm from tetramethylsilane with the solvent resonance as the internal standard $\left(\mathrm{CDCl}_{3}: 7.24 \mathrm{ppm}\right)$. Data are reported as follows: chemical shift, integration, multiplicity $(\mathrm{s}=$ singlet, $\mathrm{d}=$ doublet, $\mathrm{t}=$ triplet, $\mathrm{q}=$ quartet, $\mathrm{br}=$ broad, $\mathrm{m}=$ multiplet), coupling constants $(\mathrm{Hz})$ and assignment. ${ }^{13} \mathrm{C}$ NMR were recorded on a Varian Gemini $300(75 \mathrm{MHz})$ spectrometer with complete proton decoupling. Chemical shifts are reported in ppm from tetramethylsilane with the solvent as the internal standard $\left(\mathrm{CDCl}_{3}: 77.0\right.$ ppm). Microanalyses were preformed by Robertson Microlit.

Liquid chromatography was performed using forced flow (flash chromatography) of the indicated solvent system on Sigma silica gel $60\left(\mathrm{SiO}_{2}, 230-400 \mathrm{mesh}\right)$. Thin layer chromatography was performed on EM Science $0.25 \mathrm{~mm}$ silica gel 60 plates. Visualization was achieved with phosphomolybdic acid in ethanol followed by heating. Analytical gas-liquid chromatography (GLC) was performed on a HewlettPackard 6890 Series chromatograph equipped with a split mode capillary injection system, the indicated chiral GLC column, a flame ionization detector and using helium as the carrier gas.

All reactions were conducted in oven or flame dried glassware under an inert atmosphere of dry nitrogen. Dichloroethane was sequentially washed with concentrated $\mathrm{H}_{2} \mathrm{SO}_{4}$, water, aqueous $\mathrm{Na}_{2} \mathrm{CO}_{3}$, and water, then dried with $\mathrm{MgSO}_{4}$ and fractionally distilled from $\mathrm{CaH}_{2}$. Chloro(1,5cyclooctadiene)rhodium (I) dimer and ( $R$ )-BINAP were purchased from Strem Chemical Company. All other reagents were purchased from either Lancaster or Aldrich Chemical Companies. [(cod)RhBINAP]BF 4 was synthesized according to the procedure by: Miyshita, A.; Takaya, H.; Souchi, T.; Noyori, R. Tetrahedron 1984, 40, 1245-1253. 
Procedure for catalytic reductive aldol reaction with $(R)$-binap/[(Cod)RICl $]_{2}($ Scheme 1):

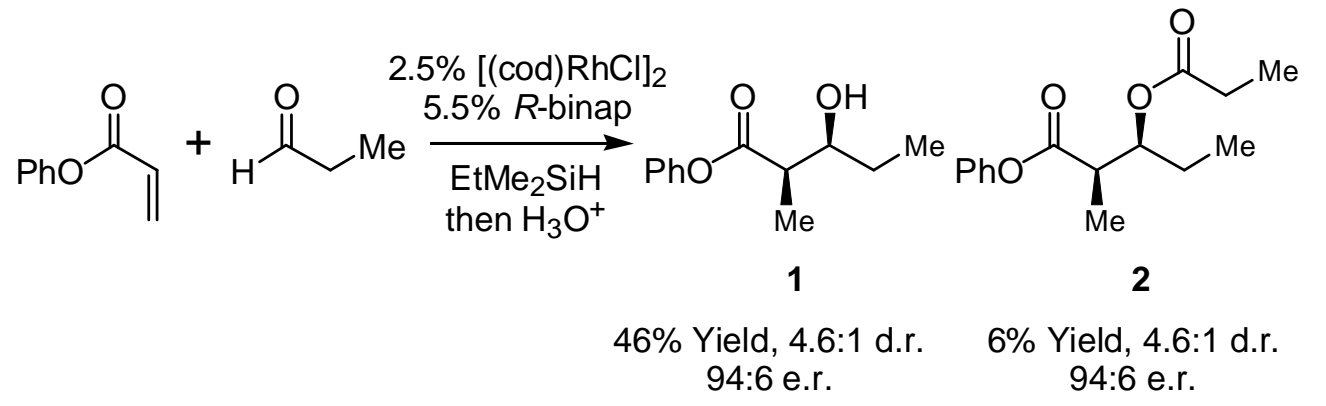

A $10 \mathrm{~mL}$ flame-dried round bottom flask was charged with $17.1 \mathrm{mg}$ [codRhCl $]_{2}(0.035 \mathrm{mmol})$ and $56 \mathrm{mg}(R)$-BINAP $(0.090 \mathrm{mmol})$ in $1.7 \mathrm{~mL}$ of 1,2-dichloroethane. The catalyst solution was stirred for 30 minute and $171 \mathrm{mg}$ of $\mathrm{Et}_{2} \mathrm{MeSiH}(1.66 \mathrm{mmol})$ added to the reaction vessel. The resulting solution was stirred at room temperature for 15 minutes. Next, $1.7 \mathrm{~mL}$ of 1,2-dichloroethane was added followed by $246 \mathrm{mg}$ phenyl acrylate $(1.66 \mathrm{mmol})$ and $81 \mathrm{mg}$ of propionaldehyde $(1.38 \mathrm{~mol})$. The vessel was then sealed and allowed to stir for 24 hours. Solvent was then evaporated from the reaction and $10 \% \mathrm{HF} / \mathrm{CH}_{3} \mathrm{CN}$ was added and the reaction stirred for $45 \mathrm{~min}$ at room temperature. Water and chloroform was then added and the water layer was extracted with chloroform ( $3 \times 25 \mathrm{~mL})$. The combined organic layers were washed with a saturated aqueous sodium chloride solution $(1 \mathrm{x} 10 \mathrm{~mL})$, dried over $\mathrm{Na}_{2} \mathrm{SO}_{4}$, and filtered. The solvent was removed by rotary evaporation to yield crude product which was used for calculation of diastereomer ratios by NMR. The crude material which was purified via flash chromatography (8:1 then 5:1 hexanes:ethyl acetate) to yield $\mathbf{1}$ and $\mathbf{2}$.

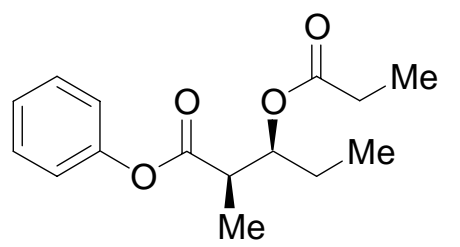

(2R,3R)-3-propionyloxy-2-methyl-pentanoic acid phenyl ester. IR (neat) $3000(\mathrm{br}, \mathrm{w}), 1756(\mathrm{~s}), 1593(\mathrm{~m}), 1193(\mathrm{~s}) \mathrm{cm}^{-1} ;{ }^{1} \mathrm{H}$ NMR: $\delta$ 7.02$7.45(5 \mathrm{H}, \mathrm{m}$, aromatic), $5.31(1 \mathrm{H}, \mathrm{m}, \mathrm{J}=7.0 \mathrm{~Hz}, \mathrm{CHCHOC}(\mathrm{O}) \mathrm{Et}), 2.99$ $\left(1 \mathrm{H}, \mathrm{m}, \mathrm{J}=7.0 \mathrm{~Hz}, \mathrm{CH}_{3} \mathrm{CH}\right), 2.42\left(2 \mathrm{H}, \mathrm{q}, \mathrm{J}=8 \mathrm{~Hz} \mathrm{CO} \mathrm{CH}_{2} \mathrm{CH}_{3}\right), 1.71$ $\left(2 \mathrm{H}, \mathrm{m}, \mathrm{CH}_{2} \mathrm{CH}_{3}\right), 1.31\left(3 \mathrm{H}, \mathrm{d}, \mathrm{J}=7.0 \mathrm{~Hz} \mathrm{CHCH}_{3}\right), 1.15(3 \mathrm{H}, \mathrm{t}, \mathrm{J}=7.0$ $\left.\mathrm{Hz}, \mathrm{CH}_{2} \mathrm{CH}_{3}\right) 0.997\left(3 \mathrm{H}, \mathrm{t}, \mathrm{J}=8.0 \mathrm{~Hz}, \mathrm{RO}_{2} \mathrm{CCH}_{2} \mathrm{CH} 3 ;{ }^{13} \mathrm{C} \mathrm{NMR}: \delta\right.$ 174.0, 150.5, 129.8, 126.3, 121.8, 75.7, 75.2, 43.6, 28.5, 25.4, 12.9, 9.7. High Resolution Mass spec for $\mathrm{C}_{15} \mathrm{H}_{21} \mathrm{O}_{4}$ : calc. $265.1440[\mathrm{M}+\mathrm{H}]^{+}$, found 265.1447.

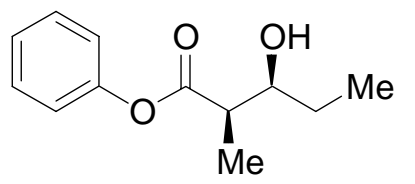

(2R,3R)-3-hydroxy-2-methylpentanoic acid phenyl ester. IR (neat) 3450 (br, s), 2980 (s), 1790 (s), 1593 (m), 1500 (m), 1200 (s) $\mathrm{cm}^{-1} ;{ }^{1} \mathrm{H}$ NMR: $\delta$ 7.02-7.37 (5H, m, aromatic), 3.98 (1H, dt, J=7.5 Hz , EtCHOH), 2.811 $\left(1 \mathrm{H}, \quad \mathrm{dq}, \mathrm{J}=7.19 \mathrm{~Hz}, \mathrm{CH}_{3} \mathrm{CH}\right), 2.41 \quad(\mathrm{EtCHOH}), 1.6 \quad(2 \mathrm{H}, \mathrm{m}$, $\left.\mathrm{CH}_{3} \mathrm{CH}_{2} \mathrm{CHOH}\right), 1.34\left(3 \mathrm{H}, \mathrm{d}, \mathrm{J}=7.0 \mathrm{~Hz}, \mathrm{CHCH}_{3}\right), 1.04(3 \mathrm{H}, \mathrm{q}, \mathrm{J}=7.0 \mathrm{~Hz}$, $\left.\mathrm{OHCHCH}_{2} \mathrm{CH}_{3}\right) .{ }^{13} \mathrm{C}$ NMR: $\delta$ 174.0, 150.5, 129.8, 126.3, 121.8, 75.7, 45.6, 29.5, 10.0. High Resolution Mass spec for $\mathrm{C}_{12} \mathrm{H}_{17} \mathrm{O}_{3}[\mathrm{M}+\mathrm{H}]^{+}$: calc. 209.1178, found 209.112. 
The enantiomeric excess for 1 and 2 from this experiment was determined by GLC analysis (Supelco, $\beta$-dex, $105^{\circ} \mathrm{C}, 15 \mathrm{psi}$ ) after conversion to 2 -methyl-1,3 pentane diol by DIBAL reduction.

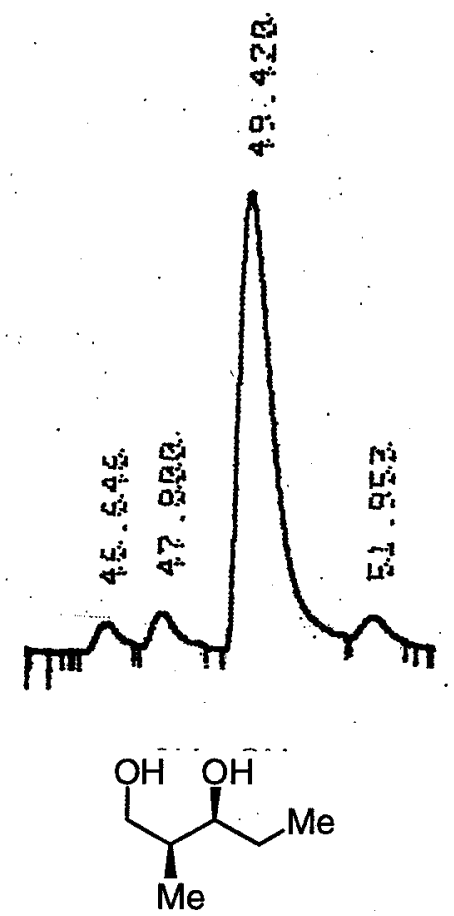

Derived from 1

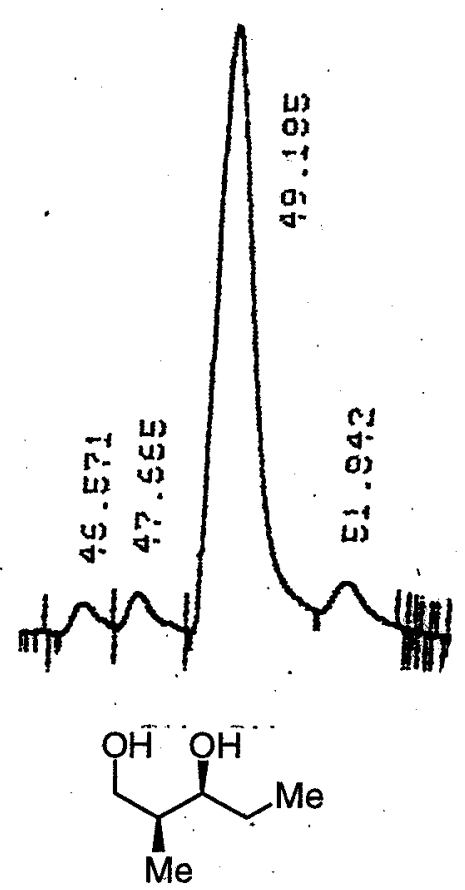

Derived from 2

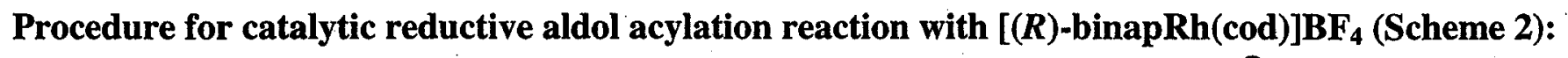<smiles>C=CC(=O)Oc1ccccc1</smiles>

Representative: A $10 \mathrm{~mL}$ flame-dried round bottom flask was charged with $64 \mathrm{mg}$ of

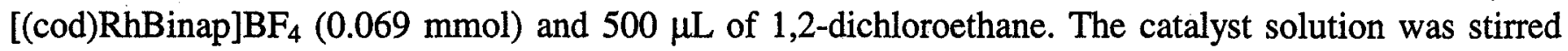
for one minute to dissolve the rhodium species, and $105 \mu \mathrm{L}$ of dimethylphenylsilane $(0.69 \mathrm{mmol})$ were added to the reaction vessel. The resulting solution was stirred at room temperature for 30 minutes. Next, $1.7 \mathrm{~mL}$ of 1,2-dichforoethane was added followed by $192 \mu \mathrm{L}$ phenyl acrylate $(1.38 \mathrm{mmol})$ and 200 $\mu \mathrm{L}$ of propionaldehyde $(2.76 \cdot \mathrm{mmol})$. The vessel was then sealed and allowed to stir for 24 hours. Solvent was evaporated from the reaction and $10 \% \mathrm{HF} / \mathrm{CH}_{3} \mathrm{CN}$ was added and the reaction stirred for $45 \mathrm{~min}$ at room temperature. Water and chloroform were then added and the water layer was extracted with chloroform $(3 \times 25 \mathrm{~mL})$. The combined organic layers were washed with a saturated aqueous sodium chloride solution ( $1 \times 10 \mathrm{~mL}$ ), dried over $\mathrm{Na}_{2} \mathrm{SO}_{4}$, and filtered. The solvent was removed by rotary evaporation to yield crude product which was purified via flash chromatography (8:1 then 5:1 hexanes:ethyl acetate) to yield $40 \mathrm{mg}$ ( $(0.27 \mathrm{mmol}, 40 \%$ yield $)$ of 2 and $25.9 \mathrm{mg}(0.12 \mathrm{mmol}, 18 \%$ yield $)$ of 1. The enantiomeric excess of each compound was determined by GLC analysis (Supelco, $\beta$-dex, $105^{\circ} \mathrm{C}, 15 \mathrm{psi}$ ) after conversion to 2-methyl-1,3 pentanediol by DIBAL reduction. 
Procedure for preparation of $\left[(R \text {-binap) } R h H]_{2}\right.$ (4) (Scheme 3):

(R-binap)Rh(cod)BF $4 \underset{\mathrm{ClCD}_{2} \mathrm{CD}_{2} \mathrm{Cl}}{\stackrel{\mathrm{PhMe}_{2} \mathrm{SiH}}{\longrightarrow}}$

3
(R-binap)Rh ${ }_{-}^{\prime} \mathrm{Hh}\left(R\right.$-binap) $+\mathrm{PhMe}_{2} \mathrm{SiF}$

4

$12.5 \mathrm{mg}$ of $\left[(\operatorname{cod}) \mathrm{Rh}(R)-\mathrm{BINAP}_{\mathrm{B}} \mathrm{BF}_{4}(0.013 \mathrm{mmol})\right.$ was weighed into an NMR tube equipped with a teflon screw cap. The tube was charged with $500 \mu \mathrm{L}$ of deuterated 1,2-dichloroethane and allowed to stand for 1 minute. $56 \mu \mathrm{L}(0.365 \mathrm{mmol})$ of phenyldimethylsilane was then added and the reaction periodically shaken for 45 minutes. ${ }^{1} \mathrm{H}$ NMR analysis of the mixture revealed an overlapping triplet of quintets at $-8.18 \mathrm{ppm}(\mathrm{J}=16.8 \mathrm{~Hz})$ which resolves to a binomial triplet $(\mathrm{J}=17 \mathrm{~Hz})$ in the $\left\{{ }^{31} \mathrm{P}\right\}{ }^{1} \mathrm{H} \mathrm{NMR}$. ${ }^{31} \mathrm{P}$ NMR revealed a doublet at $38.5 \mathrm{ppm}(\mathrm{J}=147.1 \mathrm{~Hz})$ corresponding to 4 in addition to a doublet at $25.8 \mathrm{ppm}(\mathrm{J}=145.6 \mathrm{~Hz})$ for unreacted 3. ${ }^{19} \mathrm{~F}$ NMR analysis of the catalyst mixture revealed signals corresponding to $\mathrm{PhMe}_{2} \mathrm{SiF}$ at $-161.4 \mathrm{ppm}$ (septet, $\mathrm{J}=7.5 \mathrm{~Hz},{ }^{29} \mathrm{Si}$ satellites exhibit ${ }^{1} \mathrm{~J}_{\mathrm{SiF}}=278 \mathrm{~Hz}$ ) and the precatalyst 3 at $-153.0 \mathrm{ppm}$ (singlet, shoulder at -152.9 due to ${ }^{10} \mathrm{~B}$ isotope). $\mathrm{BF}_{3}$ was not detected in the mixture.

${ }^{1} \mathrm{H}$ NMR:

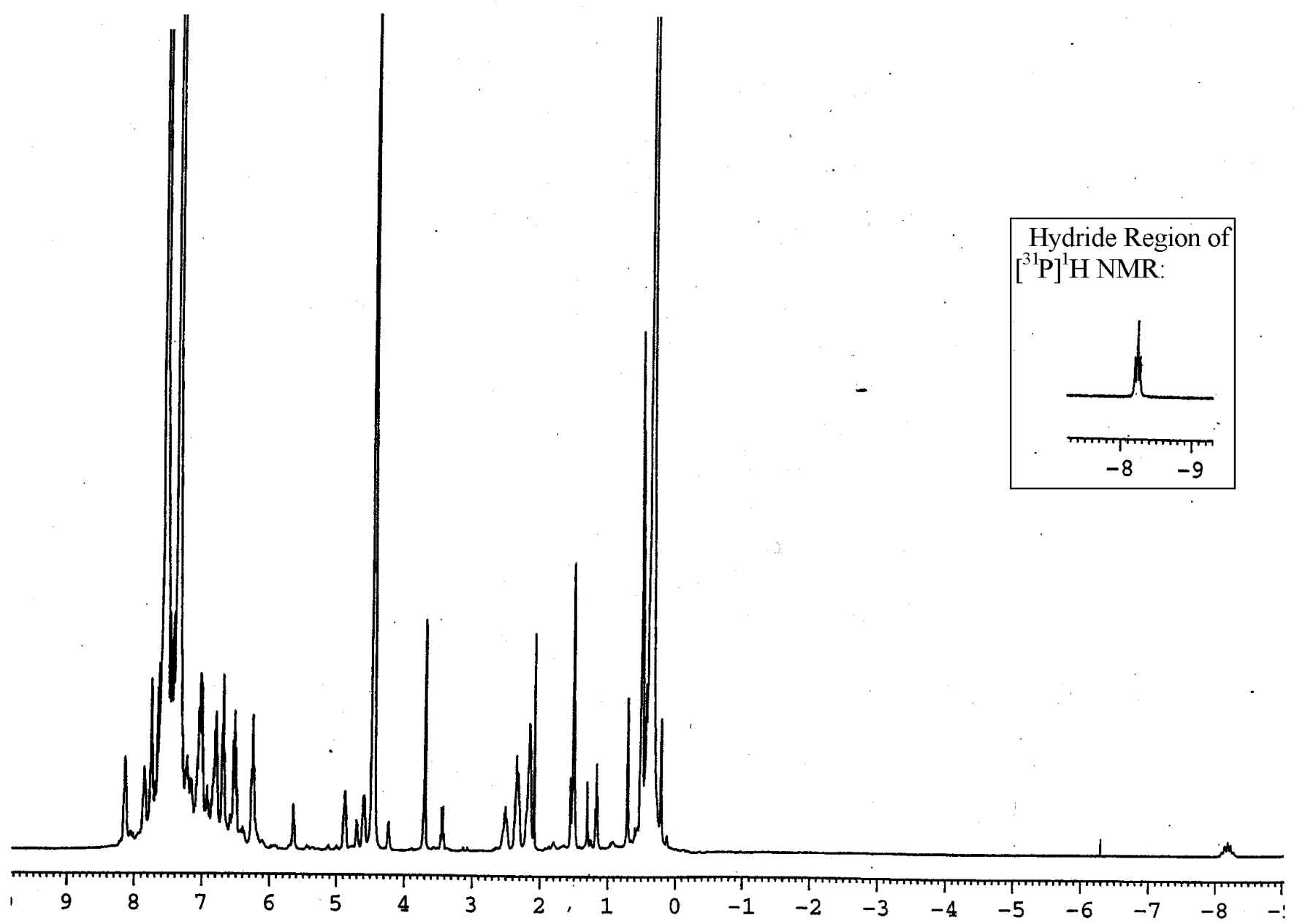



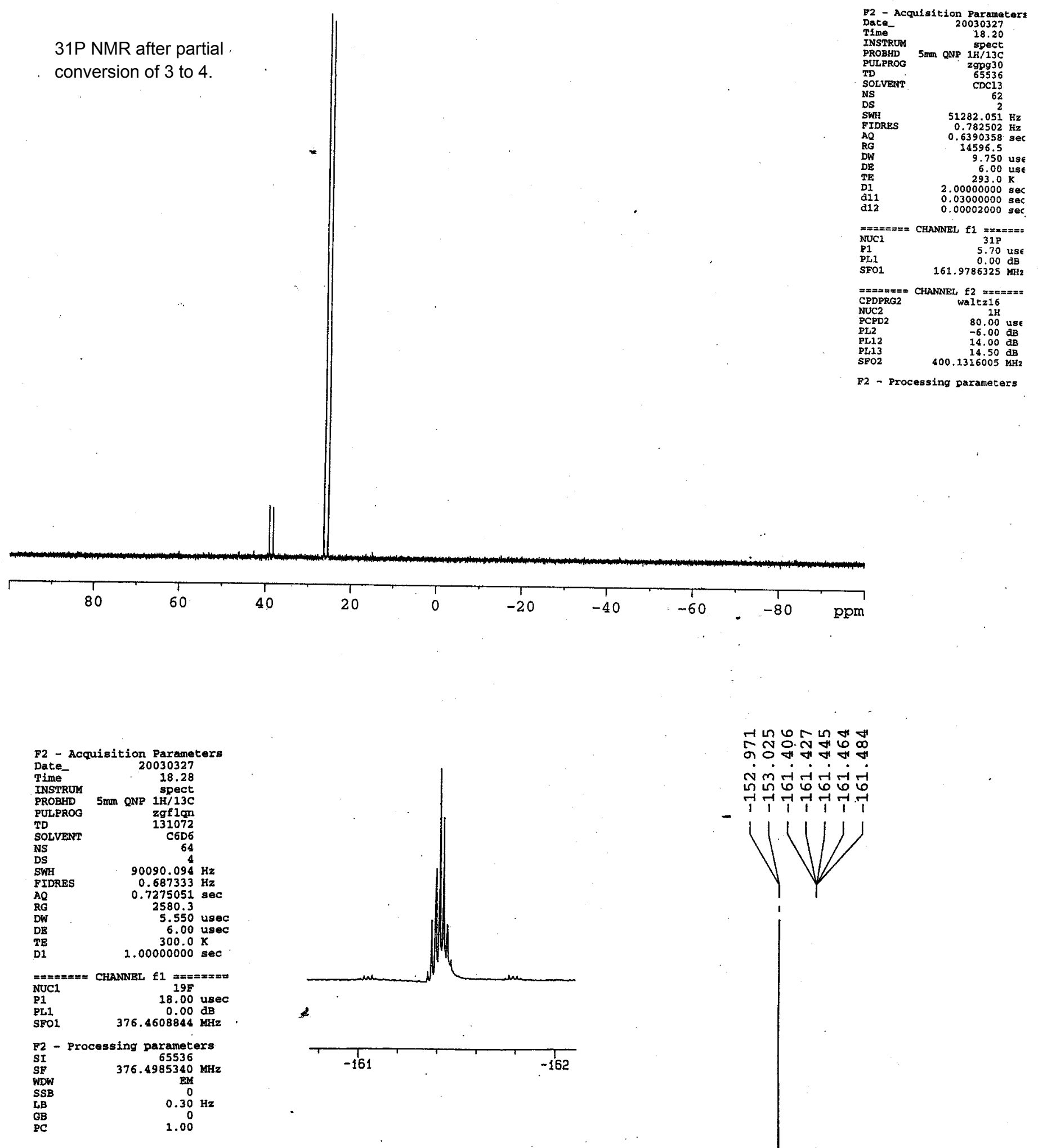

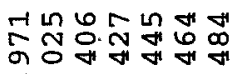

त.

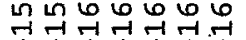
PROBHD $5 \mathrm{~mm}$ QNP $1 \mathrm{H} / 13 \mathrm{C}$ zgflgm SOLVENT NS DS FIDRES RQ DW D1 . NUC1 $\quad 18.00$ usec PL1 $\quad 0.00 \mathrm{~dB}$ F2 - Processing parameters $\begin{array}{lr}\text { SI } & 65536 \\ \text { SF } & 376.4985340 \mathrm{MHz}\end{array}$ $\begin{array}{ll}S S B & 0 \\ \text { CB } & 0.30 \mathrm{~Hz}\end{array}$

1.00
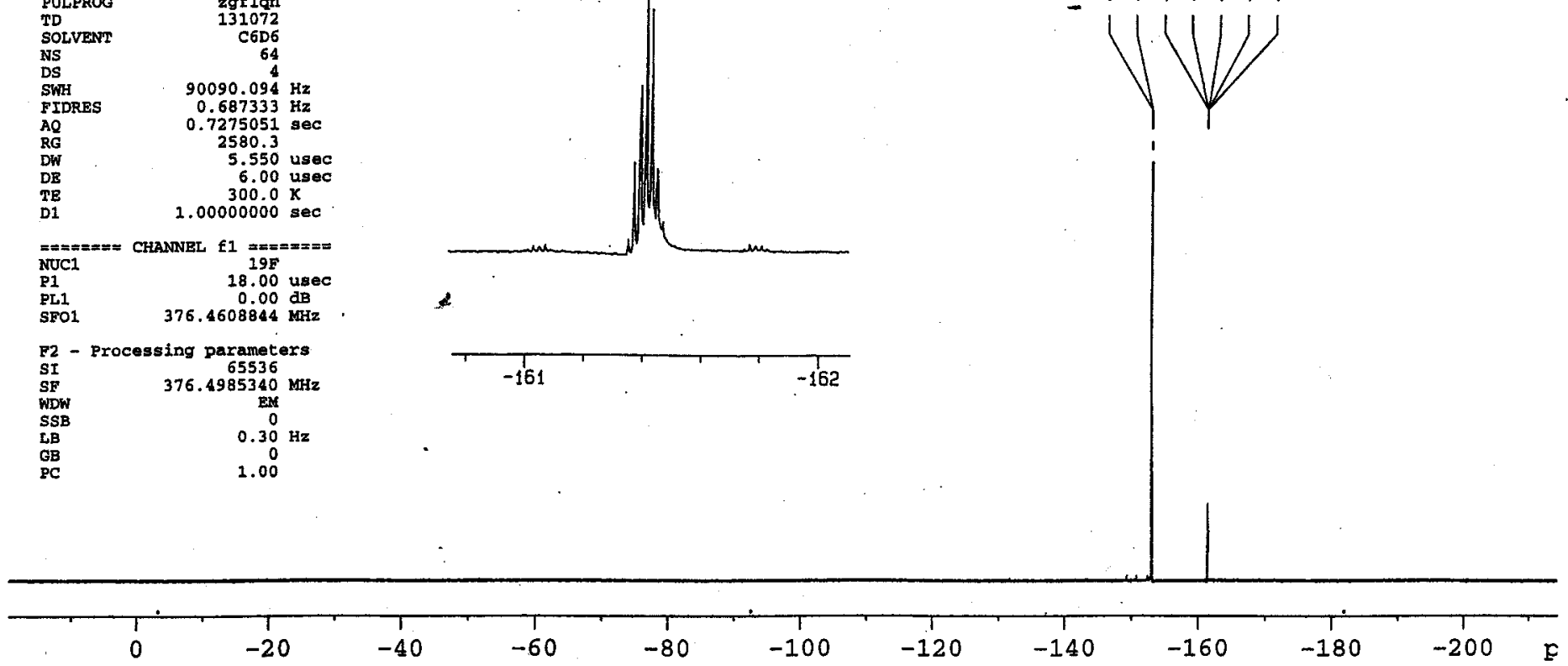
<smiles>C=CC(=O)Oc1ccccc1</smiles>

$44 \%$ yield, 3:1 d.r. $\quad 10 \%$ yield, 3:1 d.r. 86:14 e.r. $\quad 86: 14$ e.r.<smiles>O=C(OC(c1ccccc1)C(c1ccccc1)[N+](=O)[O-])c1ccccc1</smiles>

(2R,3R)-3-benzoyloxy-2-methyl-3-phenylpropionic acid phenyl ester. IR (neat) 3400 (br, w), 2900 (br, m), 1760 (s), 1720 (s), 1500 (m), 1250 (s) $\mathrm{cm}^{-1} ;{ }^{1} \mathrm{H}$ NMR: $\delta$ 6.8-8.1 (15H, m, aromatic), $6.44(1 \mathrm{H}, \mathrm{d}, \mathrm{J}=7.0 \mathrm{~Hz}, \mathrm{CHO}(\mathrm{O}) \mathrm{Ar}), 3.44(1 \mathrm{H}, \mathrm{m}$, $\left.\mathrm{CH}_{3} \mathrm{CH}\right), 1.48\left(3 \mathrm{H}, \mathrm{d}, \mathrm{J}=7.0 \mathrm{~Hz}, \mathrm{CHCH}_{3}\right) ;{ }^{13} \mathrm{C} \mathrm{NMR}: \delta 172.0,165.8,150.8,138.6$, $133.7,130.2,129.8,129.0,128.9,128.8,127.8,127.2,126.3,121.8,76.6,46.7,13.2$. HRMS Calc'd for $\mathrm{C}_{23} \mathrm{H}_{20} \mathrm{O}_{4}$ : Calc. 361.1440 , Found $361.1442[\mathrm{M}+\mathrm{H}]^{+}$.

O $\mathrm{OH}$ (2R,3R)-3-hydroxy-2-methyl-3-phenylpropionic acid phenyl ester. This compound was identical to that reported in: Taylor, S.J.; Duffey, M.O.; Morken, J.P. J. Am. Chem. Soc. 2000,122, 4528.

The enantiomeric excess of each compound was determined by GLC analysis (Supelco, $\beta$-dex, $160^{\circ} \mathrm{C}, 15 \mathrm{psi}$ ) after conversion to the derived 1,3-diol by DIBAL reduction.

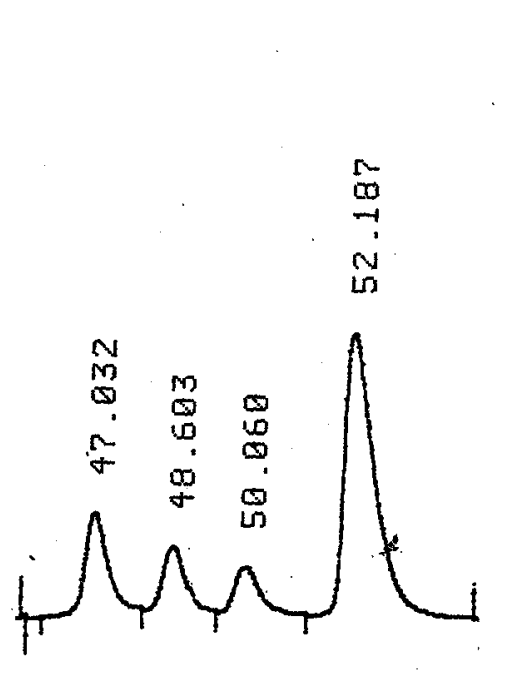<smiles>C[C@H](CO)[C@H](O)c1ccccc1</smiles>

fròm silylation adduct

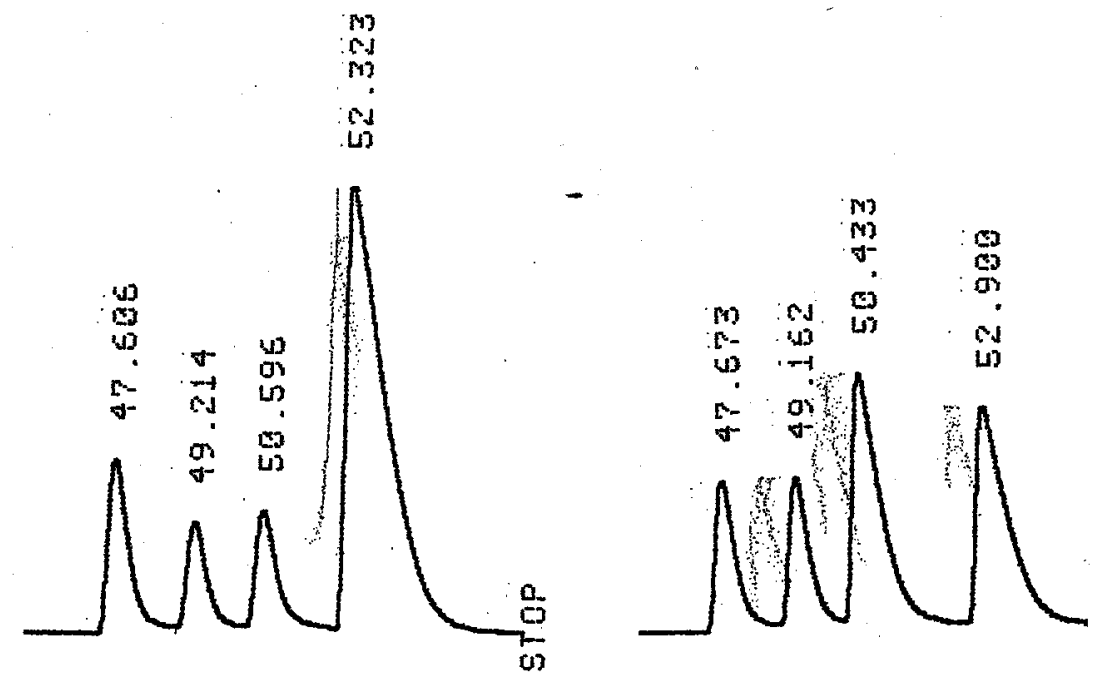<smiles>[O-][N+][C@H](O)[C@@H](O)c1ccccc1</smiles>

from acylation adduct<smiles>C[C@H](CO)[C@H](O)c1ccccc1</smiles>

racemic 
Representative procedure for catalytic reductive aldol reaction of $\alpha, \beta$-unsaturated aldehydes (Table 1): A $10 \mathrm{~mL}$ flame-dried round bottom flask was charged with $16.5 \mathrm{mg}$ of [(cod)Rh $(R)-$ binap]BF $4(0.018 \mathrm{mmol})$, and $500 \mu \mathrm{L}$ of dichloroethane. The resulting solution was stirred at room temperature for approximately one minute or until the rhodium complex was fully dissolved. After the complex was fully dissolved, $62 \mu \mathrm{L}$ of diethylmethylsilane $(0.428 \mathrm{mmol})$ was added to the mixture and the reaction vessel stirred for 30 minutes. Next, $250 \mu \mathrm{L}$ of dichloroethane followed by $50 \mu \mathrm{L}$ of phenyl acrylate $(0.357 \mathrm{mmol})$ and $172 \mu \mathrm{L} \mathrm{2-methyl-2-butenal}(1.78 \mathrm{mmol})$ was added to the solution. The vessel was then sealed and allowed to stir for 24 hours, after which time 0.5 equivalents of diethylmethylsilane $(0.1785 \mathrm{mmol})$ was added and the reaction was stirred an additional $24 \mathrm{~h}$. Solvent was then evaporated from the reaction and $1 \mathrm{~mL}$ each of $\mathrm{THF}, \mathrm{MeOH}$, and $4 \mathrm{~N} \mathrm{HCl}$ were added. This mixture was stirred at room temperature for an additional 45 minutes. Ethyl acetate was then used to extract the product $(3 \times 25 \mathrm{~mL})$. The combined organic layers were washed with a saturated aqueous sodium bicarbonate solution $(2 \times 20 \mathrm{~mL})$, dried over $\mathrm{Na}_{2} \mathrm{SO}_{4}$, and filtered. The solvent was removed by rotary evaporation to yield crude product which was purified via flash chromatography $(16: 1,14: 1$ then $13: 1$ hexanes:ethyl acetate) to yield $71 \mathrm{mg}(0.303 \mathrm{mmol}, 85 \%$ yield $)$ of a $2.5: 1$ ratio of $(2 R, 3 R)-3-$ hydroxy-2,4-dimethyl-hex-4-ene-onic acid phenyl ester (74\% ee).

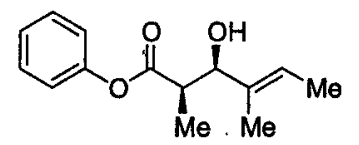

(2R,3R)-3-hydroxy-2,4-dimethyl-hex-4-ene-oic acid methyl ester. IR (KBr) 3550 (br, m) $2990(\mathrm{~m}), 1780$ (s) $1490(\mathrm{~m}), 1200(\mathrm{~s}) \mathrm{cm}^{-1}$; ${ }^{1} \mathrm{H}$ NMR: $87.5-6.98(5 \mathrm{H}$, $\mathrm{m}$, aromatic), $5.61(1 \mathrm{H}$, br q, J=6.8 Hz, vinyl CH), $4.36(1 \mathrm{H}, \mathrm{d}, \mathrm{J}=5.9 \mathrm{~Hz}, \mathrm{CHOH})$, $2.90\left(1 \mathrm{H}, \mathrm{m}, \mathrm{CH}_{3} \mathrm{CH}\right), 2.06\left(1 \mathrm{H}\right.$, br d, J=3.2 Hz, OH), $1.68\left(3 \mathrm{H}\right.$, s, vinyl $\left.\mathrm{CH}_{3}\right), 1.65\left(3 \mathrm{H}\right.$, s, vinyl $\left.\mathbf{C H}_{3}\right)$, $1.32\left(3 \mathrm{H}, \mathrm{d}, \mathrm{J}=7.0 \mathrm{~Hz}, \mathrm{CHCH}_{3}\right) ;{ }^{13} \mathrm{C}$ NMR: $\delta 174.2,151.0,135.5,129.8,126.3,122.4,121.9,78.2,43.9$, $30.1,14.8,13.5,12.4$. HRMS Calc'd for $\mathrm{C}_{14} \mathrm{H}_{19} \mathrm{O}_{3}[\mathrm{M}+\mathrm{H}]^{+}: 235.1334$, Found: 235.1323 .

Proof of Stereochemistry. Determination of stereochemical ratios was accomplished through chiral SFC analysis in comparison authentic adducts prepared by asymmetric aldol methodology (Crimmins, M.T.; Chaudhary, K. Org. Lett. 2000, 2, 775) and in comparison to non-selective aldol adducts.

Chiral SFC Analysis of Racemic and Catalytic Reaction Products.
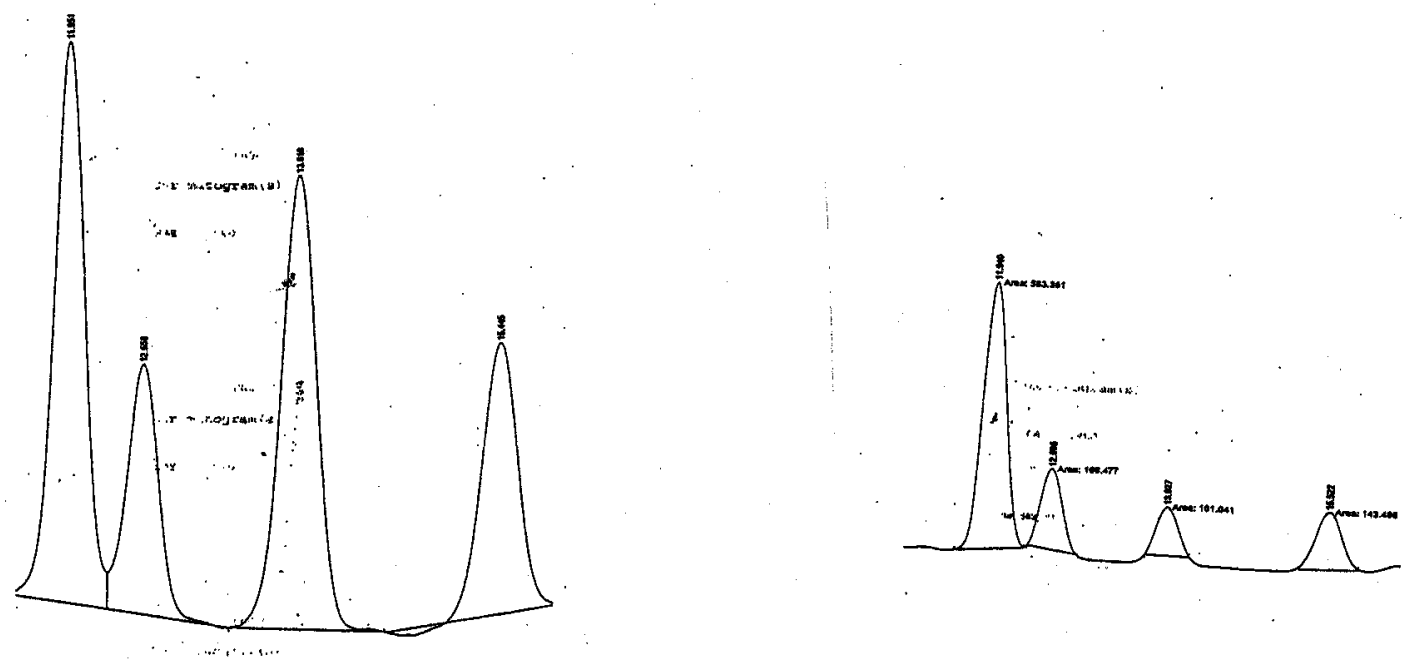
Cl

(2R,3R)-3-hydroxy-2,5-dimethyl-hex-4-ene-oic acid phenyl ester. IR (KBr) $3450(\mathrm{br}, \mathrm{s}) 2980(\mathrm{~m}), 1780(\mathrm{~s}) 1490(\mathrm{~m}), 1200(\mathrm{~s}) \mathrm{cm}^{-1} ;{ }^{1} \mathrm{H}$ NMR: $\delta$ 7.5-7.01 (5H, $\mathrm{m}$, aromatic), 5.35 (br d, J=8.0 Hz, vinylic $\mathrm{CH}) 4.71(1 \mathrm{H}, \mathrm{m}, \mathrm{CHOH}), 2.75(1 \mathrm{H}$, $\left.\mathrm{dq}, \mathrm{J}=6.7,6.8 \mathrm{~Hz}, \mathrm{CH}_{3} \mathrm{CH}\right), 2.26(1 \mathrm{H}, \mathrm{d}, \mathrm{J}=4.6 \mathrm{~Hz}, \mathrm{OH}), 1.78\left(3 \mathrm{H}, \mathrm{s}\right.$, vinyl $\left.\mathrm{CH}_{3}\right), 1.74(3 \mathrm{H}$, s, vinyl $\left.\mathrm{CH}_{3}\right), 0.88\left(3 \mathrm{H}, \mathrm{d}, \mathrm{J}=7.2 \mathrm{~Hz}, \mathrm{CHCH}_{3}\right) ;{ }^{13} \mathrm{C}$ NMR: $\delta$ 174.0, 151.0, 137.6, 129.8, 126.3, 124.8, 121.9, 70.1, 46.3, 26.3, 18.8, 12.8. HRMS Calc'd for $\mathrm{C}_{14} \mathrm{H}_{19} \mathrm{O}_{3}[\mathrm{M}+\mathrm{H}]^{+}:$235.1334, Found: 235.1342 .

Proof of Stereochemistry. Determination of stereochemical ratios was accomplished through chiral SFC analysis in comparison to non-selective aldol adducts prepared according to the method described above. Absolute configuration of the syn diastereomer was established by comparison to the authentic $2 \mathrm{~S}, 3 \mathrm{~S}$ isomer prepared by the use of a chiral auxiliary as described above.

SFC (Chiracel OD, Supelco) analysis of reductive aldol reaction product and racemic mixture of diastereomers:

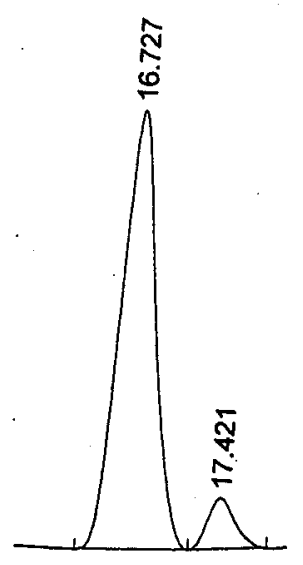

Reaction product

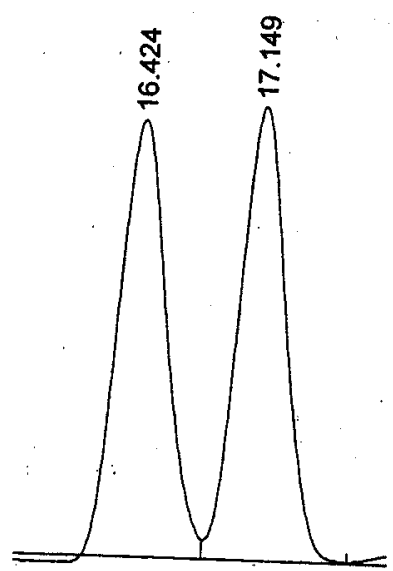

Racemic

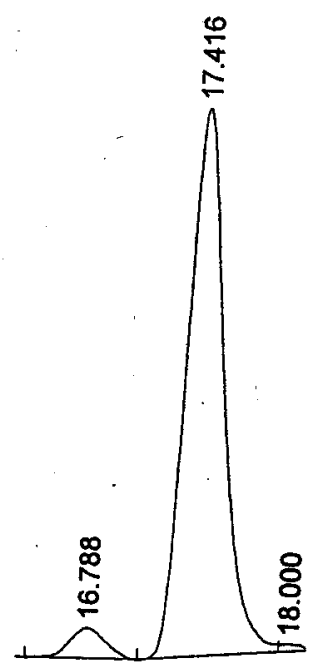

Authentic 
(N)

(2R,3R)-3-cyclohexene-3-hydroxyl-2-methylpropionic acid phenyl ester. IR (neat) $3480(\mathrm{br}, \mathrm{s}), 2950(\mathrm{~m}), 1800(\mathrm{~m}), 1800(\mathrm{~m}), 1770(\mathrm{~m}) \mathrm{cm}^{-1} ;{ }^{1} \mathrm{H}$ NMR: $\delta 7.50-$

$7.01(5 \mathrm{H}, \mathrm{m}$, aromatic), $5.80(1 \mathrm{H}, \mathrm{s}, \mathrm{CH}=\mathrm{CHOH}), 4.35(1 \mathrm{H}, \mathrm{br} \mathrm{t}, \mathrm{J}=4.5 \mathrm{~Hz}, \mathrm{CHOH})$, $2.89\left(1 \mathrm{H}, \mathrm{m}, \mathrm{CHCH}_{3}\right), 2.0-2.1(4 \mathrm{H}, \mathrm{m}$, allylic hydrogens $), 1.5-1.7(4 \mathrm{H}, \mathrm{d}, \mathrm{J}=7.8 \mathrm{~Hz}$, aliphatic), $1.3(3 \mathrm{H}$, $\left.\mathrm{d}, \mathrm{J}=7.0 \mathrm{~Hz}, \mathrm{CH}_{3}\right) ;{ }^{13} \mathrm{C}$ NMR: $\delta 174.3,151.1,137.4,129.8,126.2,124.7,121.8,77.0,43.7,25.3,24.8$, 22.9 (2 C), 12.2; HRMS Calc'd for $\mathrm{C}_{16} \mathrm{H}_{24} \mathrm{NO}_{3}\left[\mathrm{M}+\mathrm{NH}_{4}\right]^{+}: 278.1756$. Found: 278.1767 .

Proof of Stereochemistry. Determination of stereochemical ratios was accomplished through chiral SFC analysis in comparison to non-selective aldol adducts prepared according to the method described above. (2R,3R)-3-cyclohex-3-ene-3-hydroxyl-2-methylpropionic acid phenyl ester was converted to $(2 R, 3 R)$-3-cyclohexyl-3-hydroxyl-2-methylpropionic acid phenyl ester via hydrogenation with $\mathrm{H}_{2}$ and $\mathrm{PtO}_{2}$ in ethanol. The resulting compound was analyzed by SFC. Absolute configuration was established by comparison to the known $2 \mathrm{R}, 3 \mathrm{R}$ cyclohexyl reductive aldol adduct previously prepared in an asymmetric fashion (Taylor, S.J.; Duffey, M.O.; Morken, J.P. J. Am. Chem. Soc. 2000,122, 4528 ).

SFC (Chiracel OD, Supelco) analysis of reductive aldol reaction product and racemic mixture of stereoisomer:

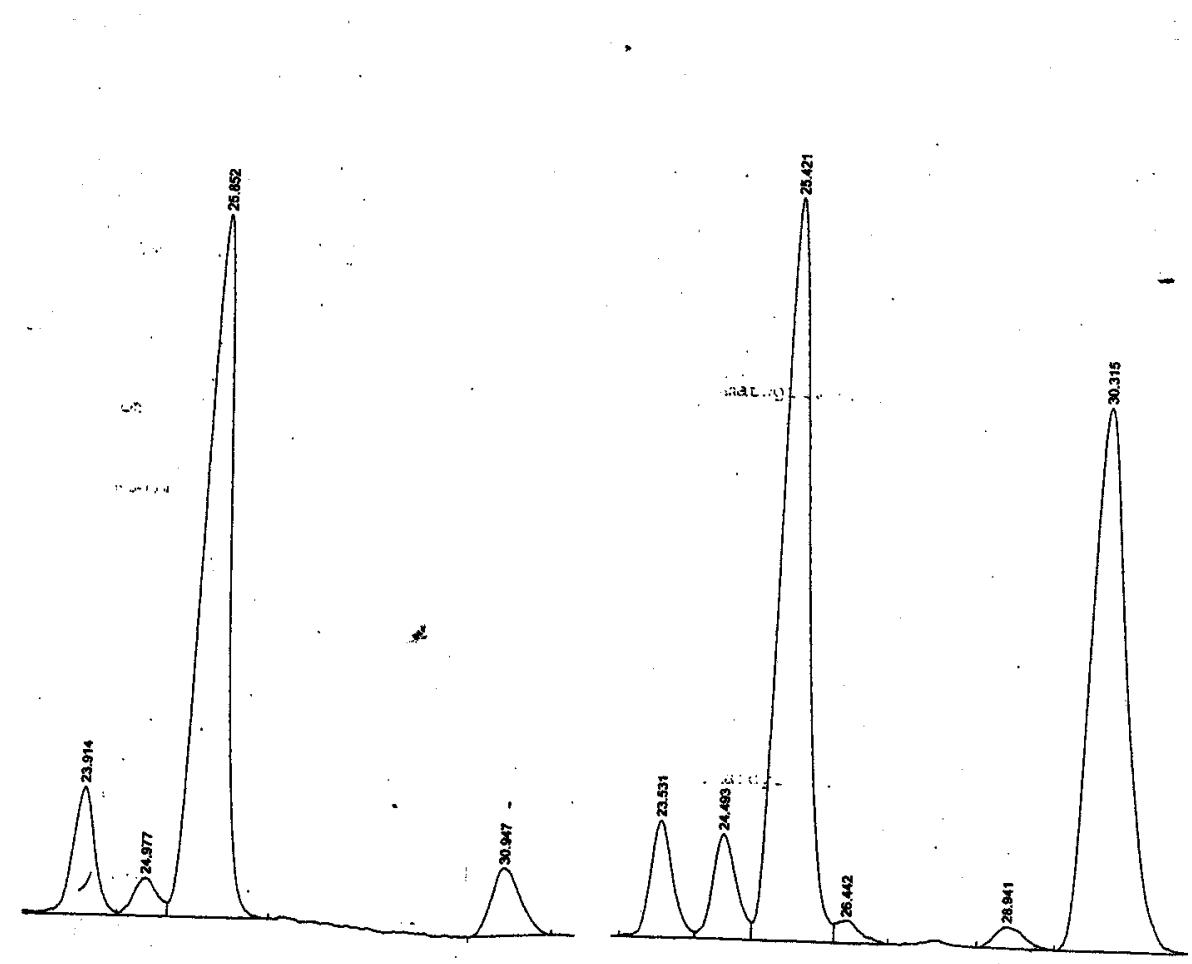

Reaction product
Racemic

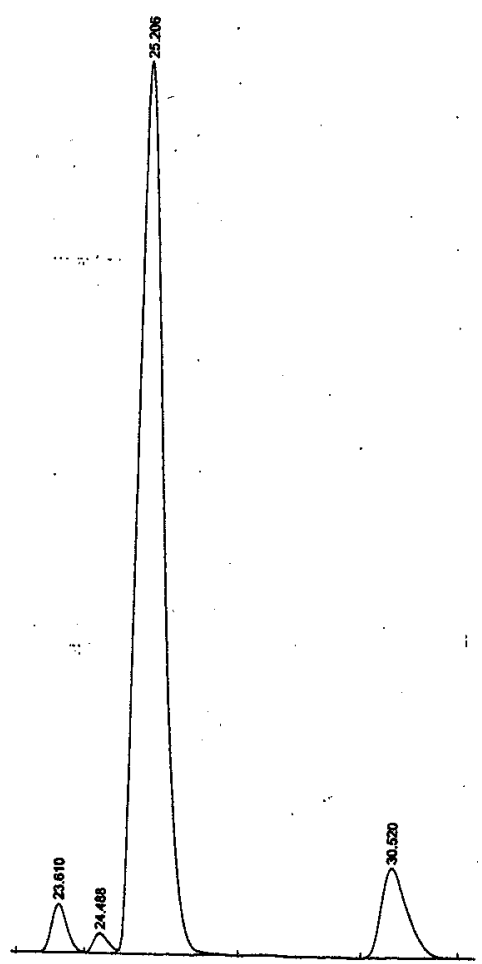

Authentic 
$12 \underbrace{O}_{M e} c_{M e}^{O H}$

(2R,3R)-3-hydroxy-2-methyl-hex-4-ene-onic acid phenyl ester. IR (KBr) 3500 $(\mathrm{br}, \mathrm{s}), 3000(\mathrm{~m}), 1780(\mathrm{~s}), 1500(\mathrm{~m}), 1200(\mathrm{~s}) \mathrm{cm}^{-1} ;{ }^{1} \mathrm{H}$ NMR: $\delta 7.5-7.03(5 \mathrm{H}, \mathrm{m}$, aromatic), $5.58(1 \mathrm{H}, \mathrm{br} \mathrm{dq}, \mathrm{J}=15.3,6.4 \mathrm{~Hz}), 5.60(1 \mathrm{H}, \mathrm{ddq}, \mathrm{J}=15.3,6.9,1.5 \mathrm{~Hz})$, $4.44(1 \mathrm{H}, \mathrm{q}, \mathrm{J}=5.1 \mathrm{~Hz}, \mathrm{CHOH}), 2.88\left(1 \mathrm{H}, \mathrm{m}, \mathrm{CH}_{3} \mathrm{CHCO}\right), 2.32(1 \mathrm{H}, \mathrm{d}, \mathrm{J}=4.5 \mathrm{~Hz}, \mathrm{OH}), 1.75(3 \mathrm{H}, \mathrm{d}$, $\left.\mathrm{J}=6.4 \mathrm{~Hz}, \mathrm{CH}=\mathrm{CHCH}_{3}\right), 1.37\left(3 \mathrm{H}, \mathrm{d}, \mathrm{J}=7.2 \mathrm{~Hz}, \mathrm{CHCH}_{3}\right) ;{ }^{13} \mathrm{C} \mathrm{NMR}: \delta 174.1,150.9,130.8,129.9,129.3$, 126.4, 121.9, 74.0, 45.9, 18.2, 12.3. HRMS Calc'd for $\mathrm{C}_{13} \mathrm{H}_{17} \mathrm{O}_{3}[\mathrm{M}+\mathrm{H}]^{+}:$221.1178, Found: 221.1177.

Proof of Stereochemistry. Stereochemical ratios were determined in comparison to racemic material and in comparison to authentic materials prepared by asymmetric aldol methodology.

Supercritical fluid chromatography (Chiralcel AS, Supelco) analysis of reductive aldol product:

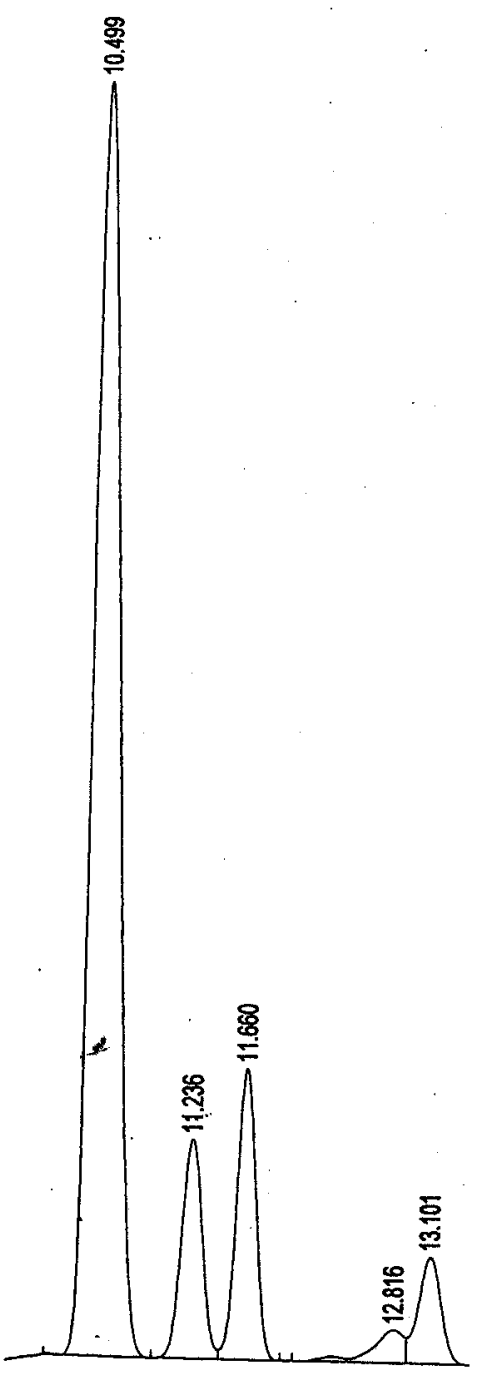

Reaction product

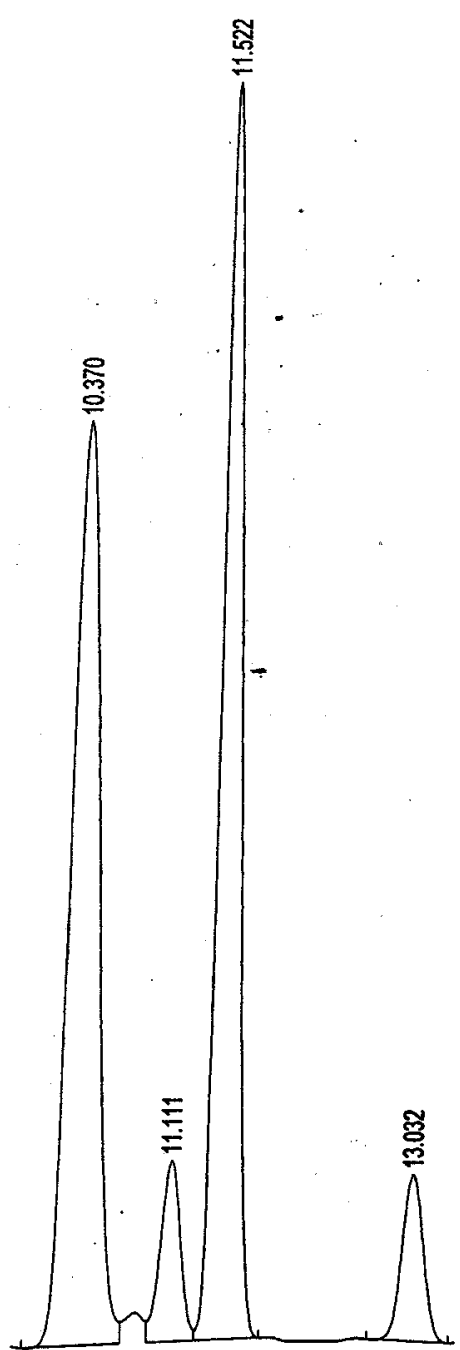

Racemic 
O Me (Z)-Phenyl Crotonate: To a flame-dried $100 \mathrm{~mL}$ flask under $\mathrm{N}_{2}$ were added 18crown-6 (3.77 g, $14.26 \mathrm{mmol})$ and $40 \mathrm{~mL}$ of dry THF. Phenylbis(trifluoroethoxy)phosphonoacetate (1.63 g, $4.28 \mathrm{mmol}$ ) was dissolved in 17 $\mathrm{mL}$ dry THF and transferred to reaction flask via syringe. The flask was then cooled to $-78{ }^{\circ} \mathrm{C}$, and potassium hexamethyldisilazide $(8.58 \mathrm{~mL}, 0.5 \mathrm{M}$ in toluene, $4.28 \mathrm{mmol})$ added dropwise via syringe. Acetaldehyde $(0.16 \mathrm{~mL}, 2.85 \mathrm{mmol})$ was added and the reaction was stirred for $45 \mathrm{~min}$ at $-78^{\circ} \mathrm{C}$. The reaction was then quenched by addition of saturated $\mathrm{NH}_{4} \mathrm{Cl}$. Extraction of the aqueous layer was done with three portions of diethyl ether. The organic layer was dried over $\mathrm{Na}_{2} \mathrm{SO}_{4}$, filtered, and concentrated. Purification by flash column chromatography in 20:1 hexane: ethyl acetate $\left(R_{f}=0.74,4: 1\right.$ hexanes:ethyl acetate), provided 0.19 grams $(31 \%)$ of pure product. ${ }^{1} \mathrm{H}$ NMR: $\delta 7.37(2 \mathrm{H}, \mathrm{t}, \mathrm{J}=7.8$ $\mathrm{Hz}, \operatorname{ArH}), 7.2(1 \mathrm{H}, \mathrm{t}, \mathrm{J}=7.7 \mathrm{~Hz}, \operatorname{ArH}), 7.1(2 \mathrm{H}, \mathrm{d}, \mathrm{J}=7.3 \mathrm{~Hz}, \operatorname{ArH}), 6.52(1 \mathrm{H}, \mathrm{dq}, \mathrm{J}=7 \mathrm{~Hz}, \mathrm{~J}=11$ $\left.\mathrm{Hz}, \mathrm{CHCH}_{3}\right), 6.02(1 \mathrm{H}, \mathrm{dq}, \mathrm{J}=10 \mathrm{~Hz}, \mathrm{~J}=2 \mathrm{~Hz}, \mathrm{C}(\mathrm{O}) \mathrm{CH}), 2.20\left(3 \mathrm{H}, \mathrm{dd}, \mathrm{J}=4.0 \mathrm{~Hz}, \mathrm{~J}=1.6 \mathrm{~Hz}, \mathrm{CH}_{3}\right)$; ${ }^{13} \mathrm{C}$ NMR: $\delta 164.7,150.7,146.7,129.3,125.5,122.1,121.6,15.5$.

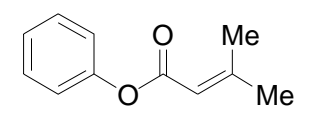

3-Methyl-but-2-enoic Acid Phenyl Ester: To a flame-dried $250 \mathrm{~mL}$ flask under $\mathrm{N}_{2}$ were added phenol (7.94 g, $\left.84 \mathrm{mmol}\right)$ and dry $\mathrm{CH}_{2} \mathrm{Cl}_{2}(84 \mathrm{~mL})$, and the mixture was cooled to $0{ }^{\circ} \mathrm{C}$. Triethylamine $(23.5 \mathrm{~mL}, 168 \mathrm{mmol})$ was added and the reaction mixture was stirred at $0{ }^{\circ} \mathrm{C}$ for 45 minutes. After addition of 3,3-dimethyl-acryloyl chloride (84 mmol), a bright yellow mixture formed which was stirred at $0{ }^{\circ} \mathrm{C}$ for $1 \mathrm{~h}$, then warmed to ambient temperature and stirred overnight. The reaction was quenched with $1 \mathrm{M} \mathrm{HCl}$, and the organic and aqueous layers were separated. The organic layer was washed with saturated $\mathrm{NaHCO}_{3}$ and brine, then dried over $\mathrm{MgSO}_{4}$, filtered, and concentrated. Purification via column chromatography in 8:1 hexanes:ethyl acetate $\left(R_{f}=0.73,4: 1\right.$ hexanes:ethyl acetate) provided $5.98 \mathrm{~g}(80 \%)$ of the desired ester. ${ }^{1} \mathrm{H}$ NMR: $\delta 7.35(2 \mathrm{H}, \mathrm{t}, \mathrm{J}=8.2 \mathrm{~Hz}, \operatorname{ArH}), 7.2(1 \mathrm{H}, \mathrm{t}, \mathrm{J}=7.4 \mathrm{~Hz}, \operatorname{ArH}), 7.08(2 \mathrm{H}, \mathrm{d}, \mathrm{J}=7.5 \mathrm{~Hz}, \operatorname{ArH})$, $5.90(1 \mathrm{H}, \mathrm{s}, \mathrm{C}(\mathrm{O}) \mathrm{CH}), 2.21\left(3 \mathrm{H}, \mathrm{s}, \mathrm{CCH}_{3}\right), 1.97\left(3 \mathrm{H}, \mathrm{s}, \mathrm{CCH}_{3}\right) ;{ }^{13} \mathrm{C} \mathrm{NMR}: \delta 164.8,159.7,150.7$, $129.3,125.4,121.8,115.3,27.6,20.4$.

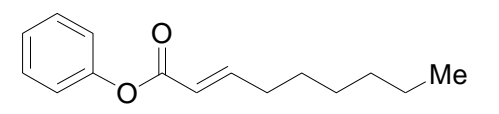

Phenyl 2-nonenoate. $\operatorname{LiBr}(0.45 \mathrm{~g}, 5.16 \mathrm{mmol})$ was weighed into a dry $25 \mathrm{~mL}$ flask under $\mathrm{N}_{2}$. Then a solution of phenyl diethylphosphonoacetate $(1.17 \mathrm{~g}, 4.3 \mathrm{mmol})$ in dry THF $(4.3 \mathrm{~mL})$ was added via syringe. The mixture was stirred for $5 \mathrm{~min}$, then triethylamine was added $(0.66 \mathrm{~mL}, 4.73$ mmol). After stirring for $10 \mathrm{~min}$ heptaldehyde $(0.6 \mathrm{~mL}, 4.3 \mathrm{mmol})$ was added dropwise and the reaction allowed to stir overnight at ambient temperature. The reaction was quenched by addition of $1 \mathrm{M} \mathrm{HCl}$ and the mixture extracted with three portions of diethyl ether. The organic layer was dried over $\mathrm{Na}_{2} \mathrm{SO}_{4}$, filtered, and concentrated. Purification by column chromatography in 10:1 hexanes:ethyl acetate $\left(R_{f}=\right.$ $0.41)$, provided $0.49 \mathrm{~g}(50 \%)$ of the desired ester. ${ }^{1} \mathrm{H} \mathrm{NMR}: \delta 7.36(2 \mathrm{H}, \mathrm{t}, \mathrm{J}=8 \mathrm{~Hz}, \operatorname{ArH}), 7.23(1 \mathrm{H}, \mathrm{t}$, $\mathrm{J}=7.4 \mathrm{~Hz}, \operatorname{ArH}), 7.16\left(1 \mathrm{H}, \mathrm{dt}, \mathrm{J}=15.6 \mathrm{~Hz}, \mathrm{~J}=8.6 \mathrm{~Hz}, \mathrm{CHCHCH}_{2}\right), 7.10(2 \mathrm{H}, \mathrm{d}, \mathrm{J}=7.5 \mathrm{~Hz}, \operatorname{ArH})$, $6.00(1 \mathrm{H}, \mathrm{dt}, \mathrm{J}=15.6 \mathrm{~Hz}, \mathrm{~J}=1.5 \mathrm{~Hz}, \mathrm{C}(\mathrm{O}) \mathrm{CH}), 2.26\left(2 \mathrm{H}, \mathrm{dq}, \mathrm{J}=7.1 \mathrm{~Hz}, \mathrm{~J}=1.5 \mathrm{~Hz}, \mathrm{CHCH}_{2} \mathrm{CH}_{2}\right)$, $1.50\left(2 \mathrm{H}, \mathrm{m}, \mathrm{CHCH}_{2} \mathrm{CH}_{2}\right), 1.30\left(6 \mathrm{H}, \mathrm{m}, \mathrm{CH}_{2} \mathrm{CH}_{2} \mathrm{CH}_{2} \mathrm{CH}_{3}\right), 0.88(3 \mathrm{H}, \mathrm{t}, \mathrm{J}=6.8 \mathrm{~Hz}, \mathrm{CH}) ;{ }^{13} \mathrm{C} \mathrm{NMR:}$ $\delta 165.5,152.4,151.2,129.8,126.1,122.1,120.9,32.8,32.0,29.3,28.3,23.0,14.5$.

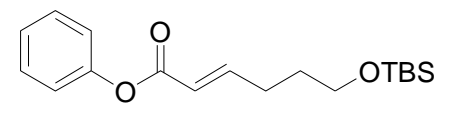

6-(tert-Butyl-dimethyl-silanoxy)-hex-2-enoic Acid Phenyl Ester. To a flame-dried $10 \mathrm{~mL}$ flask were added (triphenyl- $\lambda^{5}$-phosphanylidene)acetic acid phenyl ester (1.38 g, $3.5 \mathrm{mmol})$, 4-(tert-butyl-dimethylsilanoxy)-butyraldehyde $(0.47 \mathrm{~g}, 2.33 \mathrm{mmol})$, and dry $\mathrm{CH}_{2} \mathrm{Cl}_{2}(5.8 \mathrm{~mL})$. The flask was fit with a reflux condenser and heated at reflux for $16 \mathrm{~h}$, at which point it was cooled to RT, and the solvent was removed. The crude reaction mixture was directly purified by silica gel chromatography with 5:1 
hexanes:ethyl acetate as eluent $\left(R_{f}=0.61\right)$, yielding $0.55 \mathrm{~g}$ of the ester $(74 \%) .{ }^{1} \mathrm{H}$ NMR: $\delta 7.36(2 \mathrm{H}, \mathrm{t}$, $\mathrm{J}=8.0 \mathrm{~Hz}, \operatorname{ArH}), 7.23-7.15(3 \mathrm{H}, \mathrm{m}, 2 \mathrm{ArH}, \mathrm{C}(\mathrm{O}) \mathrm{CHCH}), 7.10$ (2 H, m, ArH), $6.02(1 \mathrm{H}, \mathrm{d}, \mathrm{J}=15.6$ $\mathrm{Hz}, \mathrm{C}(\mathrm{O}) \mathrm{CH}), 3.65\left(2 \mathrm{H}, \mathrm{t}, \mathrm{J}=6.4 \mathrm{~Hz}, \mathrm{CH}_{2} \mathrm{OSi}\right.$ ), 2.35 (2 H, q, J = 6.7 Hz, CHCHCH ), 1.71 ( $2 \mathrm{H}$, quin, $\left.\mathrm{J}=6.8 \mathrm{~Hz}, \mathrm{CH}_{2} \mathrm{CH}_{2} \mathrm{O}\right), 0.89\left(9 \mathrm{H}, \mathrm{s},\left(\mathrm{CH}_{3}\right)_{3} \mathrm{CSi}\right), 0.05\left(6 \mathrm{H}, \mathrm{s},\left(\mathrm{CH}_{3}\right)_{2} \mathrm{Si}\right) ;{ }^{13} \mathrm{C} \mathrm{NMR:} \delta 165.3,151.8$, $151.1,129.7,126.0,122.1,121.1,63.0,62.5,31.4,29.3,26.4,18.7,-4.9$.

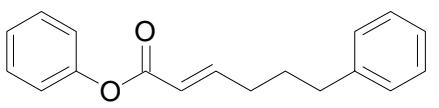

6-Phenyl-hex-2-enoic Acid Phenyl Ester. $\mathrm{LiBr}(0.57 \mathrm{~g}, 6.55 \mathrm{mmol})$ was weihed into a dry $25 \mathrm{~mL}$ flask under $\mathrm{N}_{2}$. A solution of phenyl diethylphosphonoacetate $(1.48 \mathrm{~g}, 5.46 \mathrm{mmol})$ in dry THF $(5.5 \mathrm{~mL})$ was added via syringe. After stirring for $5 \mathrm{~min}$ triethylamine $(0.84 \mathrm{~mL}, 6.0 \mathrm{mmol})$ was added. After stirring for another $10 \mathrm{~min}$, 4-phenylbutyraldehyde was added dropwise and the reaction allowed to stir at room temperature for $16 \mathrm{~h}$. The reaction was quenched by addition of $1 \mathrm{M} \mathrm{HCl}$, then the mixture was extracted with three portions of diethyl ether. The organic layer was dried over $\mathrm{MgSO}_{4}$, filtered, and concentrated. Purification by flash column chromatography in 10:1 hexanes:ethyl acetate $\left(R_{f}=0.38\right)$, provided $0.31 \mathrm{~g}(22 \%)$ of the ester. ${ }^{1} \mathrm{H}$ NMR: $\delta 7.37(2 \mathrm{H}, \mathrm{t}, \mathrm{J}=8.0 \mathrm{~Hz}, \operatorname{ArH}), 7.28(2 \mathrm{H}, \mathrm{m}, \operatorname{ArH}), 7.23$ - $7.08(7 \mathrm{H}, \mathrm{m}, 6 \mathrm{ArH}, \mathrm{C}(\mathrm{O}) \mathrm{CHCH}), 6.02(1 \mathrm{H}, \mathrm{dt}, \mathrm{J}=7.2 \mathrm{~Hz}, \mathrm{~J}=1.6 \mathrm{~Hz}, \mathrm{C}(\mathrm{O}) \mathrm{CH}), 2.67(2 \mathrm{H}, \mathrm{t}, \mathrm{J}=$ $\left.7.7 \mathrm{~Hz}, \mathrm{ArCH}_{2}\right), 2.30\left(2 \mathrm{H}, \mathrm{q}, \mathrm{J}=6.8, \mathrm{CHCHCH}_{2}\right), 1.84\left(2 \mathrm{H}\right.$, quin, $\left.\mathrm{J}=7.6 \mathrm{~Hz}, \mathrm{CHCH}_{2} \mathrm{CH}_{2}\right)$; ${ }^{13} \mathrm{C} \mathrm{NMR}$ : $\delta 165.4,151.6,141.9,129.7,128.9,128.8,128.7,126.4,126.0,122.0,121.3,35.6,32.2,29.9$.

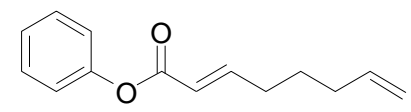

Octa-2,7-dienoic Acid Phenyl Ester. Charged a flame-dried $50 \mathrm{~mL}$ flask with (triphenyl- $\lambda^{5}$-phosphanylidene)-acetic acid phenyl ester (4.4 g, 13.7 mmol), hex-5-enal $(0.90 \mathrm{~g}, 9.17 \mathrm{mmol})$, and dry $\mathrm{CH}_{2} \mathrm{Cl}_{2}(23 \mathrm{~mL})$. The flask was fitted with reflux condenser and heated at reflux for $16 \mathrm{~h}$. After cooling to room temperature the solvent was removed and directly purified by silica gel chromatography using 5:1 hexanes:ethyl acetate $\left(R_{f}=0.74\right)$, resulting in isolation of $1.08 \mathrm{~g}(54 \%)$ of the desired product. ${ }^{1} \mathrm{H}$ NMR: $\delta 7.52(2 \mathrm{H}, \mathrm{t}, \mathrm{J}=$ $8.0 \mathrm{~Hz}, \mathrm{ArH}), 7.39$ - 7.23 (4 H, m, $3 \mathrm{ArH}, \mathrm{C}(\mathrm{O}) \mathrm{CHCH}), 6.19(1 \mathrm{H}, \mathrm{dt}, \mathrm{J}=15.6 \mathrm{~Hz}, \mathrm{~J}=1.6 \mathrm{~Hz}$, $\mathrm{C}(\mathrm{O}) \mathrm{CH}), 5.98\left(1 \mathrm{H}, \mathrm{ddt}, \mathrm{J}=17.0 \mathrm{~Hz}, \mathrm{~J}=10.2 \mathrm{~Hz}, \mathrm{~J}=6.6 \mathrm{~Hz}, \mathrm{C}_{3} \mathrm{H}_{6} \mathrm{CHCH}_{2}\right), 5.16(2 \mathrm{H}, \mathrm{m}$, $\left.\mathrm{C}_{3} \mathrm{H}_{6} \mathrm{CHCH}_{2}\right), 2.45\left(2 \mathrm{H}, \mathrm{q}, \mathrm{J}=7.6 \mathrm{~Hz}, \mathrm{CHCHCH}_{2}\right), 2.27\left(2 \mathrm{H}, \mathrm{q}, \mathrm{J}=7.1 \mathrm{~Hz}, \mathrm{C}_{2} \mathrm{H}_{4} \mathrm{CH}_{2} \mathrm{CHCH}_{2}\right), 1.77$ (2 $\mathrm{H}$, quin, $\left.\mathrm{J}=7.6 \mathrm{~Hz}, \mathrm{CHCHCH}_{2} \mathrm{CH}_{2}\right) ;{ }^{13} \mathrm{C} \mathrm{NMR}: \delta 151.2,150.8,137.8,129.3,125.6,121.6,121.5$, $120.8,115.2,33.0,31.6,27.0$.

Representative Procedure for Catalytic, Enantioselective Reductive Aldol Reaction of Crotonate Derivatives (Table 2). In an inert atmosphere glovebox, an oven-dried $250 \mathrm{~mL}$ flask equipped with a stir bar was charged with $0.37 \mathrm{~g}(0.76 \mathrm{mmol})$ of chloro(1,5-cyclooctadiene)rhodium (I) dimer, $1.21 \mathrm{~g}$ $(1.98 \mathrm{mmol})$ of $(S)$-BINAP, and $30 \mathrm{~mL}$ dry dichloroethane under an argon atmosphere. After stirring for one hour, diethylmethylsilane $(21.7 \mathrm{~mL}, 5.00 \mathrm{mmol})$ was added and the reaction mixture was stirred for $30 \mathrm{~min}$. Propionaldehyde $(2.2 \mathrm{~mL}, 31 \mathrm{mmol})$, phenyl crotonate $(5.5 \mathrm{~mL}, 36 \mathrm{mmol})$, and $30 \mathrm{~mL}$ of dry dichloroethane were then added, and the reaction was stirred at ambient temperature for $48 \mathrm{~h}$. After 48 $\mathrm{h}$, the solvent was removed by rotary evaporation, and the crude mixture was stirred with a 1:1:1 THF:MeOH:4 N HCl mixture $(39 \mathrm{~mL})$ for $1 \mathrm{~h}$. Water $(30 \mathrm{~mL})$ was then added and the layers separated. The aqueous layer was extracted with ethyl acetate three times, and then the combined organic layers were washed twice with saturated $\mathrm{NaHCO}_{3}$, dried over $\mathrm{Na}_{2} \mathrm{SO}_{4}$, filtered, and concentrated. The product was purified by flash column chromatography in 4:1 hexanes:ethyl acetate $\left(R_{f}=0.12,7: 1\right.$ hexanes: ethyl acetate), providing $5.13 \mathrm{~g}(76 \%)$ of 2-ethyl-3-hydroxy-pentanoic acid phenyl ester in a 4.3:1 syn:anti ratio of diastereomers ( $88 \%$ ee for the $s y n$ diastereomer). 


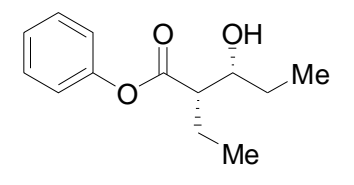

2-Ethyl-3-hydroxy-pentanoic acid phenyl ester. IR (neat): 3446 (br, s), 1756 (s), 1646 (s), 1594 (s), 1495 (s), 1383 (s), 1349 (s) cm ${ }^{-1} ;{ }^{1} \mathrm{H}$ NMR: $\delta 7.53$ (2 H, t, $\mathrm{J}=7.8 \mathrm{~Hz}, \operatorname{ArH}), 7.38(1 \mathrm{H}, \mathrm{t}, \mathrm{J}=7.4 \mathrm{~Hz}, \operatorname{ArH}), 7.22(2 \mathrm{H}, \mathrm{d}, \mathrm{J}=7.0 \mathrm{~Hz}, \operatorname{ArH})$, $4.13(1 \mathrm{H}, \mathrm{m}, \mathrm{CHOH}), 2.79(1 \mathrm{H}, \mathrm{m}, \mathrm{C}(\mathrm{O}) \mathrm{CH}), 2.33(1 \mathrm{H}, \mathrm{d}, \mathrm{J}=5.0 \mathrm{~Hz}, \mathrm{OH})$, $2.06-1.9\left(2 \mathrm{H}, \mathrm{m}, \mathrm{C}(\mathrm{O}) \mathrm{CHCH}_{2}\right), 1.8-1.7\left(2 \mathrm{H}, \mathrm{m}, \mathrm{CHCHCH} \mathbf{H}_{2}\right), 1.23(3 \mathrm{H}, \mathrm{t}, \mathrm{J}=7.5 \mathrm{~Hz}$, $\left.\mathrm{C}(\mathrm{O}) \mathrm{CHCH}_{2} \mathrm{CH}_{3}\right), 1.18\left(3 \mathrm{H}, \mathrm{t}, \mathrm{J}=7.4 \mathrm{~Hz}, \mathrm{CHCHCH}_{2} \mathrm{CH}_{3}\right) ;{ }^{13} \mathrm{C} \mathrm{NMR}: \delta 173.7,150.4,129.4,125.9$, 121.5, 73.5, 52.7, 27.5, 20.6, 12.1, 10.2. HRMS (FAB) Calc'd for $\mathrm{C}_{13} \mathrm{H}_{18} \mathrm{O}_{3}(\mathrm{M}+\mathrm{Na})^{+}$: 245.1148 . Found $(\mathrm{M}+\mathrm{Na})^{+}: 245.1152$.

${ }^{1} \mathrm{H}$ NMR of the anti diastereomer: $\delta 7.53(2 \mathrm{H}, \mathrm{t}, \mathrm{J}=7.8 \mathrm{~Hz}, \operatorname{ArH}), 7.38(1 \mathrm{H}, \mathrm{t}, \mathrm{J}=7.4 \mathrm{~Hz}, \operatorname{ArH}), 7.22$ $(2 \mathrm{H}, \mathrm{d}, \mathrm{J}=7.0 \mathrm{~Hz}, \mathrm{ArH}), 3.90(1 \mathrm{H}, \mathrm{m}, \mathrm{CHOH}), 2.79(1 \mathrm{H}, \mathrm{m}, \mathrm{C}(\mathrm{O}) \mathrm{CH}), 2.46(1 \mathrm{H}, \mathrm{d}, \mathrm{J}=8.0 \mathrm{~Hz}, \mathrm{OH})$, $2.06-1.9\left(2 \mathrm{H}, \mathrm{m}, \mathrm{C}(\mathrm{O}) \mathrm{CHCH}_{2}\right), 1.8-1.7\left(2 \mathrm{H}, \mathrm{m}, \mathrm{CHCHCH}_{2}\right), 1.23(3 \mathrm{H}, \mathrm{t}, \mathrm{J}=7.5 \mathrm{~Hz}$, $\left.\mathrm{C}(\mathrm{O}) \mathrm{CHCH}_{2} \mathrm{CH}_{3}\right), 1.18\left(3 \mathrm{H}, \mathrm{t}, \mathrm{J}=7.4 \mathrm{~Hz}, \mathrm{CHCHCH}_{2} \mathrm{CH}_{3}\right)$.

Proof of Stereochemistry: Stereochemical ratios were determined in comparison to racemic materials prepared via the reductive aldol reaction using racemic-BINAP as ligand. Absolute stereochemistry established in comparison to authentic $2(S), 3(R)$ isomer prepared via a Crimmins aldol reaction using the thiazolidinethione butyrate to provide the appropriate Evans-syn aldol product (Chaudhary, $\mathrm{K}$ and Crimmins, M. T. Org. Lett. 2000, 2, 775).

Chiral capillary electrophoresis $\left(5 \% \gamma\right.$-cyclodextrin, $\left.-15 \mathrm{kV}, 20^{\circ} \mathrm{C}\right)$ analysis of reductive aldol product.

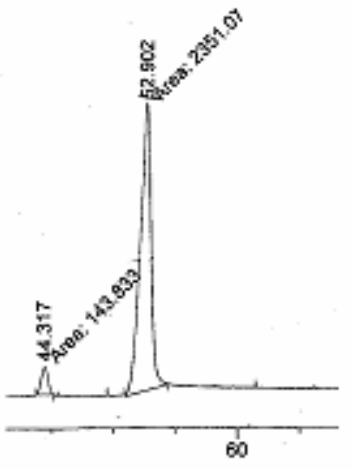

Reductive Aldol Product

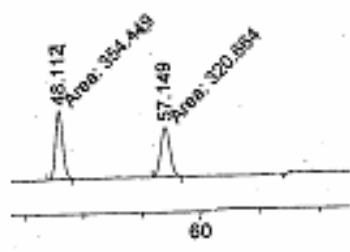

Racemic Aldol Product

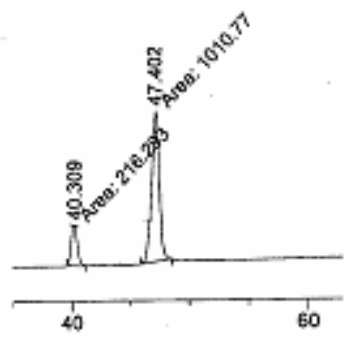

Racemic + Reductive Aldol Product Coinjection 


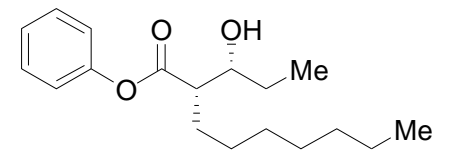

2-(1-Hydroxy-propyl)-nonanoic acid phenyl ester: IR (neat): 3577 (br, d), 1756 (s), 1652 (s), $989(\mathrm{~s}), 567(\mathrm{~m}) \mathrm{cm}^{-1} ;{ }^{1} \mathrm{H}$ NMR: $\delta 7.39(2 \mathrm{H}, \mathrm{t}, \mathrm{J}=$ $7.6 \mathrm{~Hz}, \operatorname{ArH}), 7.22(1 \mathrm{H}, \mathrm{t}, \mathrm{J}=8.0 \mathrm{~Hz}, \operatorname{ArH}), 7.05(2 \mathrm{H}, \mathrm{d}, \mathrm{J}=7.6 \mathrm{~Hz}$, ArH), 3.85 (1 H, m, CHOH), 2.67 (1 H, m, C(O)CH), $2.19(1 \mathrm{H}$, br s, OH), $1.90-1.20$ (14 H, m, methylene protons), $1.02\left(3 \mathrm{H}, \mathrm{t}, \mathrm{J}=7.4 \mathrm{~Hz}, \mathrm{CHCH}_{2} \mathrm{CH}_{3}\right), 0.86(3 \mathrm{H}, \mathrm{t}, \mathrm{J}=$ $\left.6.9 \mathrm{~Hz}, \mathrm{CH}_{2} \mathrm{CH}_{2} \mathrm{CH}_{3}\right) ;{ }^{13} \mathrm{C}$ NMR: $\delta$ 174.4, 150.8, 126.5, 121.8, 74.1, 73.9, 51.1, 50.9, 32.2, 29.9, 29.5, 28.2, 27.8, 23.0, 14.5, 10.7. HRMS (FAB) Calc'd for $\mathrm{C}_{18} \mathrm{H}_{28} \mathrm{O}_{3}(\mathrm{M}+\mathrm{Na})^{+}$: 315.1931. Found $(\mathrm{M}+$ $\mathrm{Na})^{+}: 315.1919$.

${ }^{1} \mathrm{H}$ NMR of the anti diastereomer: $\delta 7.39(2 \mathrm{H}, \mathrm{t}, \mathrm{J}=7.6 \mathrm{~Hz}, \operatorname{ArH}), 7.22(1 \mathrm{H}, \mathrm{t}, \mathrm{J}=8.0 \mathrm{~Hz}, \operatorname{ArH}), 7.05$ $(2 \mathrm{H}, \mathrm{d}, \mathrm{J}=7.6 \mathrm{~Hz}, \mathrm{ArH}), 3.70(1 \mathrm{H}, \mathrm{m}, \mathrm{CHOH}), 2.67(1 \mathrm{H}, \mathrm{m}, \mathrm{C}(\mathrm{O}) \mathrm{CH}), 2.29(1 \mathrm{H}, \mathrm{d}, \mathrm{J}=8.0 \mathrm{~Hz}, \mathrm{OH})$, $1.90-1.20$ (14 H, m, methylene protons), $1.02\left(3 \mathrm{H}, \mathrm{t}, \mathrm{J}=7.4 \mathrm{~Hz}, \mathrm{CHCH}_{2} \mathrm{CH}_{3}\right), 0.86(3 \mathrm{H}, \mathrm{t}, \mathrm{J}=6.9 \mathrm{~Hz}$, $\left.\mathrm{CH}_{2} \mathrm{CH}_{2} \mathrm{CH}_{3}\right)$.

Chiral GLC ( $\beta$-dex, Supelco) analysis of diols prepared by DIBAL reduction of reductive aldol products:

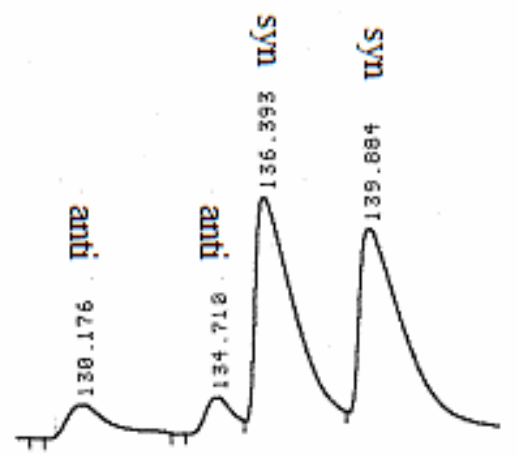

Racemic Product

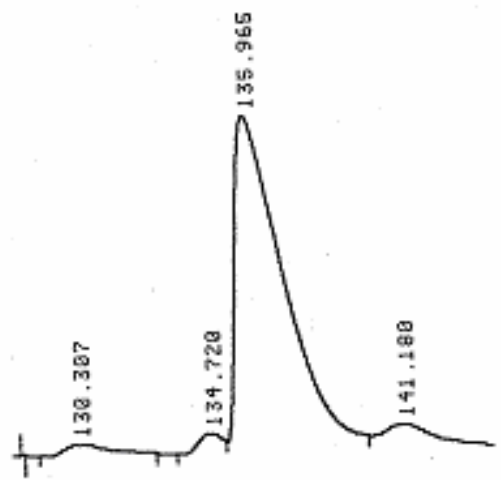

Reductive Aldol Product

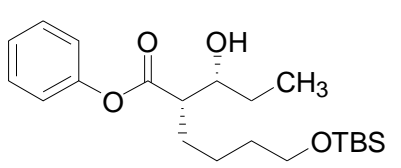

6-(tert-Butyl-dimethylsilanoxy)-2-(1-hydroxy-propyl)-hexanoic phenyl ester. IR (neat): 3566 (br, d), $1756(\mathrm{~s}), 1646(\mathrm{~s}), 559(\mathrm{~m}) \mathrm{cm}^{-1},{ }^{1} \mathrm{H}$ NMR: $\delta 7.52(2 \mathrm{H}, \mathrm{t}, \mathrm{J}=8.3 \mathrm{~Hz}, \operatorname{ArH}), 7.38(1 \mathrm{H}, \mathrm{t}, \mathrm{J}=7.5 \mathrm{~Hz}, \operatorname{ArH}), 7.21$ $(2 \mathrm{H}, \mathrm{d}, \mathrm{J}=7.5 \mathrm{~Hz}, \operatorname{ArH}), 4.01(1 \mathrm{H}, \mathrm{q}, \mathrm{J}=6.8 \mathrm{~Hz}, \mathrm{CHOH}), 3.78(2 \mathrm{H}, \mathrm{t}, \mathrm{J}=$ $\left.6.2 \mathrm{~Hz}, \mathrm{CH}_{2} \mathrm{OSi}\right), 2.84(1 \mathrm{H}, \mathrm{m}, \mathrm{C}(\mathrm{O}) \mathrm{CH}), 2.39$ (1 H, br s, OH), $2.10-1.85(2 \mathrm{H}, \mathrm{m}, \mathrm{C}(\mathrm{O}) \mathrm{CHCH}$ ), 1.8 - $1.6\left(6 \mathrm{H}, \mathrm{m}, \mathrm{CH}_{2} \mathrm{CH}_{2} \mathrm{CH}_{2} \mathrm{OSi}, \mathrm{C}(\mathrm{O}) \mathrm{CHCHCH}_{2}\right), 1.18\left(3 \mathrm{H}, \mathrm{t}, \mathrm{J}=7.4 \mathrm{~Hz}, \mathrm{CH}_{2} \mathrm{CH}_{3}\right), 1.04(9 \mathrm{H}, \mathrm{s}$, $\left.\left(\mathrm{CH}_{3}\right)_{3} \mathrm{CSi}\right), 0.18\left(6 \mathrm{H}, \mathrm{s},\left(\mathrm{CH}_{3}\right)_{2} \mathrm{Si}\right) ;{ }^{13} \mathrm{C} \mathrm{NMR:} \delta 173.8,150.4,129.4,125.9,121.5,73.6,62.8,51.0$, 32.7, 27.4, 27.1, 25.9, 24.1, 18.3, 10.2, 5.3. HRMS (FAB) Calc'd for $\mathrm{C}_{21} \mathrm{H}_{36} \mathrm{SiO}_{4}(\mathrm{M}+\mathrm{Na})^{+}:$403.2275. Found $(\mathrm{M}+\mathrm{Na})^{+}: 403.2268$.

${ }^{1} \mathrm{H}$ NMR of the anti diastereomer: $\delta 7.52(2 \mathrm{H}, \mathrm{t}, \mathrm{J}=8.3 \mathrm{~Hz}, \operatorname{ArH}), 7.38(1 \mathrm{H}, \mathrm{t}, \mathrm{J}=7.5 \mathrm{~Hz}, \operatorname{ArH}), 7.21$ $(2 \mathrm{H}, \mathrm{d}, \mathrm{J}=7.5 \mathrm{~Hz}, \mathrm{ArH}), 3.88(1 \mathrm{H}, \mathrm{q}, \mathrm{J}=6.8 \mathrm{~Hz}, \mathrm{CHOH}), 3.78\left(2 \mathrm{H}, \mathrm{t}, \mathrm{J}=6.2 \mathrm{~Hz}, \mathrm{CH}_{2} \mathrm{OSi}\right), 2.84$ (1 $\mathrm{H}, \mathrm{m}, \mathrm{C}(\mathrm{O}) \mathrm{CH}), 2.39$ (1 H, br s, OH), 2.10 - 1.85 (2 H, m, C(O)CHCH $\mathbf{H}_{2}, 1.8$ - $1.6(6 \mathrm{H}, \mathrm{m}$, $\left.\mathrm{CH}_{2} \mathrm{CH}_{2} \mathrm{CH}_{2} \mathrm{OSi}, \mathrm{C}(\mathrm{O}) \mathrm{CHCHCH}_{2}\right), 1.18\left(3 \mathrm{H}, \mathrm{t}, \mathrm{J}=7.4 \mathrm{~Hz}, \mathrm{CH}_{2} \mathrm{CH}_{3}\right), 1.04\left(9 \mathrm{H}, \mathrm{s},\left(\mathrm{CH}_{3}\right)_{3} \mathrm{CSi}\right), 0.18$ (6 $\left.\mathrm{H}, \mathrm{s},\left(\mathrm{CH}_{3}\right)_{2} \mathrm{Si}\right)$. 
Chiral capillary electrophoresis $\left(5 \% \beta\right.$-cyclodextrin, $\left.-15 \mathrm{kV}, 10^{\circ} \mathrm{C}\right)$ analysis of the diol resulting from deprotection of the silyl ether:

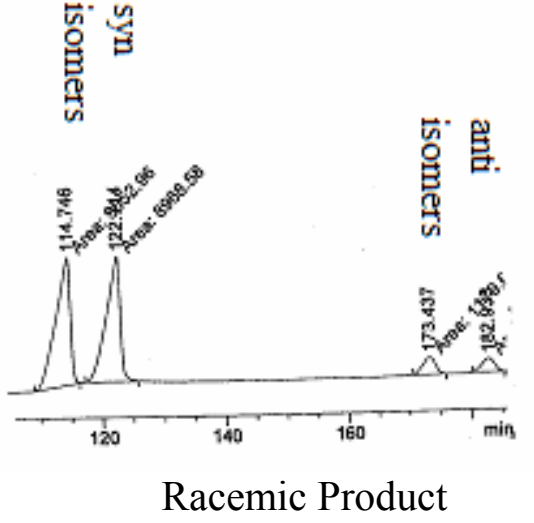

Racemic Product

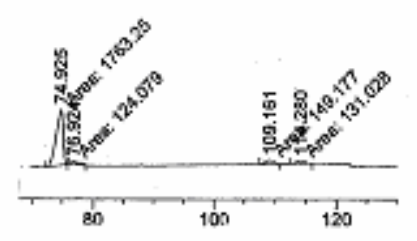

Reductive Aldol Product

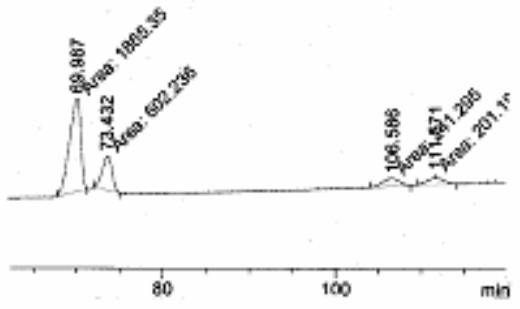

Coinjection

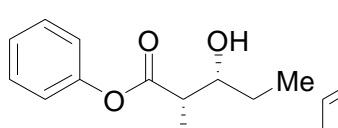

2-(1-Hydroxy-propyl)-6-phenyl-hexanoic acid phenyl ester: IR (neat): 3566 (br, d), 1756 (s), 1646 (s), 1594 (s), 1495 (s), 989 (br, s), 559 (m) cm ; ${ }^{1} \mathrm{H}$ NMR: $\delta 7.52(2 \mathrm{H}, \mathrm{t}, \mathrm{J}=8.9 \mathrm{~Hz}, \operatorname{ArH}), 7.42(3 \mathrm{H}, \mathrm{m}, \operatorname{ArH}), 7.33(3 \mathrm{H}$, m, ArH), 7.13 (2 H, m, ArH), 4.02 (1 H, m, CHOH), 2.83 (1 H, m, $\mathrm{C}(\mathrm{O}) \mathrm{CH}), 2.80\left(2 \mathrm{H}, \mathrm{t}, \mathrm{J}=7.6 \mathrm{~Hz}, \mathrm{CH}_{2} \mathrm{Ar}\right), 2.35(1 \mathrm{H}$, br s, OH $), 2.10-1.55(8 \mathrm{H}, \mathrm{m}$, $\left.\mathrm{CH}_{2} \mathrm{CH}_{2} \mathrm{CH}_{2} \mathrm{CH}_{2} \mathrm{Ar}, \mathrm{CHCH}_{2} \mathrm{CH}_{3}\right), 1.18\left(3 \mathrm{H}, \mathrm{t}, \mathrm{J}=7.4 \mathrm{~Hz}, \mathrm{CH}_{3}\right) ;{ }^{13} \mathrm{C} \mathrm{NMR:} \delta 174.3,150.7,142.7$, 129.8, 128.8, 128.7, 126.4, 126.1, 121.9, 74.0, 51.3, 36.0, 31.7, 27.9, 27.7, 27.5, 10.6. HRMS (FAB) Calc'd for $\mathrm{C}_{21} \mathrm{H}_{26} \mathrm{O}_{3}(\mathrm{M}+\mathrm{Na})^{+}: 349.1774$. Found $(\mathrm{M}+\mathrm{Na})^{+}: 349.1778$.

${ }^{1} \mathrm{H}$ NMR of the anti diastereomer: $\delta 7.52(2 \mathrm{H}, \mathrm{t}, \mathrm{J}=8.9 \mathrm{~Hz}, \operatorname{ArH}), 7.42(3 \mathrm{H}, \mathrm{m}, \operatorname{ArH}), 7.33(3 \mathrm{H}, \mathrm{m}$, ArH), 7.13 (2 H, m, ArH), 3.87 (1 H, m, CHOH), $2.83(1 \mathrm{H}, \mathrm{m}, \mathrm{C}(\mathrm{O}) \mathrm{CH}), 2.80(2 \mathrm{H}, \mathrm{t}, \mathrm{J}=7.6 \mathrm{~Hz}$, $\left.\mathrm{CH}_{2} \mathrm{Ar}\right), 2.45(1 \mathrm{H}, \mathrm{d}, \mathrm{J}=8.0 \mathrm{~Hz}, \mathrm{OH}), 2.10-1.55\left(8 \mathrm{H}, \mathrm{m}, \mathrm{CH}_{2} \mathrm{CH}_{2} \mathrm{CH}_{2} \mathrm{CH}_{2} \mathrm{Ar}, \mathrm{CHCH}_{2} \mathrm{CH}_{3}\right), 1.18$ (3 $\left.\mathrm{H}, \mathrm{t}, \mathrm{J}=7.4 \mathrm{~Hz}, \mathrm{CH}_{3}\right)$.

Supercritical fluid chromatography $\left(\mathrm{AD}-\mathrm{H}, 150 \mathrm{psi}, 50^{\circ} \mathrm{C}\right.$, flow $\left.=3 \mathrm{~mL} / \mathrm{min}, 3 \% \mathrm{MeOH}\right)$ analysis of the reductive aldol product:

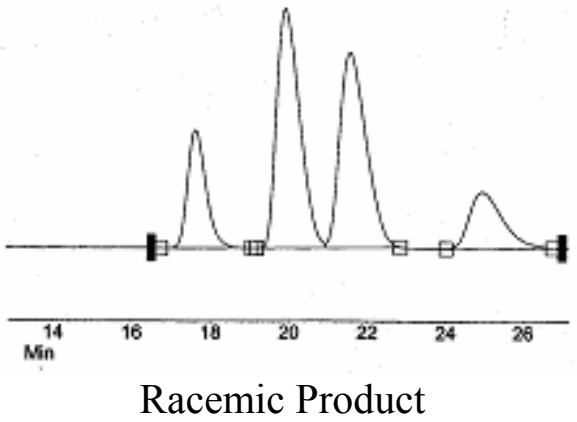

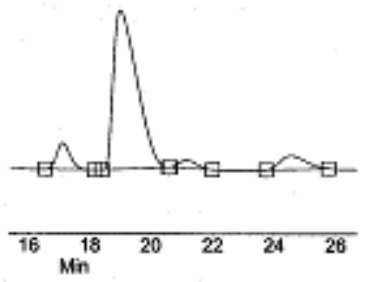

Reductive Aldol Product

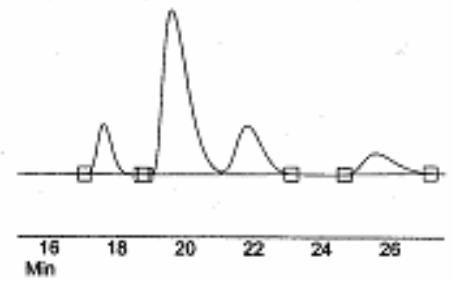

Coinjection 


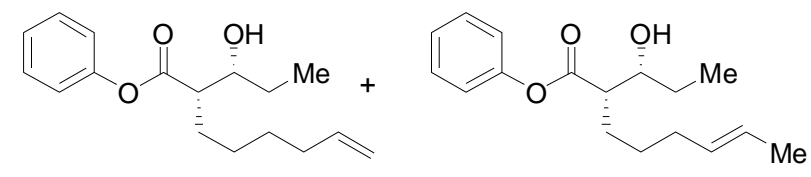

2-(1-Hydroxy-proply)-oct-7-enoic acid phenyl ester: Product obtained as a 1:1.8 mixture of reductive aldol product and olefin isomerized reductive aldol product. IR (neat): $3566(\mathrm{br}, \mathrm{d})$, 1756 (s), 1717 (s), 1652 (s), 1461 (s), 989 (s), 542 (m) cm ${ }^{-1} ;{ }^{1} \mathrm{H}$ NMR: $\delta 7.53(2 \mathrm{H}, \mathrm{t}, \mathrm{J}=7.9 \mathrm{~Hz}, \operatorname{ArH})$, $7.38(1 \mathrm{H}, \mathrm{t}, \mathrm{J}=7.5 \mathrm{~Hz}, \mathrm{ArH}), 7.21(2 \mathrm{H}, \mathrm{d}, \mathrm{J}=8.5 \mathrm{~Hz}, \mathrm{ArH}), 5.95\left(1 \mathrm{H}, \mathrm{m}, \mathrm{CH}_{2} \mathrm{CHCH}_{2}\right), 5.12(2 \mathrm{H}, \mathrm{m}$, $\left.\mathrm{CH}_{2} \mathrm{CH}_{2} \mathrm{CHCH}_{2}\right), 4.01(1 \mathrm{H}, \mathrm{m}, \mathrm{CHOH}), 2.84(1 \mathrm{H}, \mathrm{m}, \mathrm{C}(\mathrm{O}) \mathrm{CH}), 2.34(1 \mathrm{H}$, br s, OH $), 2.23(2 \mathrm{H}, \mathrm{m}$, $\left.\mathrm{CH}_{2} \mathrm{CH}_{2} \mathrm{CHCH}_{2}\right), 2.10-1.80\left(2 \mathrm{H}, \mathrm{m}, \mathrm{C}(\mathrm{O}) \mathrm{CHCH}_{2}\right), 1.80-1.55\left(6 \mathrm{H}, \mathrm{C}(\mathrm{O}) \mathrm{CHCH}_{2} \mathrm{CH}_{2} \mathrm{CH}_{2}\right.$, $\left.\mathrm{CHCH}_{2} \mathrm{CH}_{3}\right), 1.18\left(3 \mathrm{H}, \mathrm{t}, \mathrm{J}=7.4 \mathrm{~Hz}, \mathrm{CH}_{2} \mathrm{CH}_{3}\right) ;{ }^{13} \mathrm{C} \mathrm{NMR}: \delta 173.8,150.4,129.4,125.9,121.5,114.5$, 73.6, 51.0, 33.4, 32.4, 28.7, 27.6, 26.7, 17.8, 10.2. HRMS (FAB) Calc'd for $\mathrm{C}_{17} \mathrm{H}_{24} \mathrm{O}_{3}(\mathrm{M}+\mathrm{Na})^{+}$: 299.1618. Found $(\mathrm{M}+\mathrm{Na})^{+}: 299.1630$.

${ }^{1} \mathrm{H}$ NMR of the anti diastereomer: $\delta 7.53(2 \mathrm{H}, \mathrm{t}, \mathrm{J}=7.9 \mathrm{~Hz}, \operatorname{ArH}), 7.38(1 \mathrm{H}, \mathrm{t}, \mathrm{J}=7.5 \mathrm{~Hz}, \operatorname{ArH}), 7.21$ $(2 \mathrm{H}, \mathrm{d}, \mathrm{J}=8.5 \mathrm{~Hz}, \mathrm{ArH}), 5.95\left(1 \mathrm{H}, \mathrm{m}, \mathrm{CH}_{2} \mathrm{CHCH}_{2}\right), 5.12\left(2 \mathrm{H}, \mathrm{m}, \mathrm{CH}_{2} \mathrm{CH}_{2} \mathrm{CHCH}_{2}\right), 3.87(1 \mathrm{H}, \mathrm{m}$, $\mathrm{CHOH}), 2.84(1 \mathrm{H}, \mathrm{m}, \mathrm{C}(\mathrm{O}) \mathrm{CH}), 2.45(1 \mathrm{H}, \mathrm{d}, \mathrm{J}=8.0 \mathrm{~Hz}, \mathrm{OH}), 2.23\left(2 \mathrm{H}, \mathrm{m}, \mathrm{CH}_{2} \mathrm{CH}_{2} \mathrm{CHCH}_{2}\right), 2.10-$ $1.80\left(2 \mathrm{H}, \mathrm{m}, \mathrm{C}(\mathrm{O}) \mathrm{CHCH}_{2}\right), 1.80-1.55\left(6 \mathrm{H}, \mathrm{C}(\mathrm{O}) \mathrm{CHCH}_{2} \mathrm{CH}_{2} \mathrm{CH}_{2}, \mathrm{CHCH}_{2} \mathrm{CH}_{3}\right), 1.18(3 \mathrm{H}, \mathrm{t}, \mathrm{J}=7.4$ $\left.\mathrm{Hz}, \mathrm{CH}_{2} \mathrm{CH}_{3}\right)$.

${ }^{1} \mathrm{H}$ NMR of the internal olefin product: $\delta 7.53(2 \mathrm{H}, \mathrm{t}, \mathrm{J}=7.9 \mathrm{~Hz}, \mathrm{ArH}), 7.38(1 \mathrm{H}, \mathrm{t}, \mathrm{J}=7.5 \mathrm{~Hz}, \mathrm{ArH})$, $7.21(2 \mathrm{H}, \mathrm{d}, \mathrm{J}=8.5 \mathrm{~Hz}, \mathrm{ArH}), 5.62\left(2 \mathrm{H}, \mathrm{m}, \mathrm{CH}_{2} \mathbf{C H C H C H}_{3}\right), 4.01(1 \mathrm{H}, \mathrm{m}, \mathrm{CHOH}), 2.84(1 \mathrm{H}, \mathrm{m}$, $\mathrm{C}(\mathrm{O}) \mathrm{CH}), 2.34(1 \mathrm{H}, \mathrm{br} \mathrm{s}, \mathrm{OH}), 2.23\left(2 \mathrm{H}, \mathrm{m}, \mathrm{CH}_{2} \mathrm{CHCHCH}_{3}\right), 2.10-1.80\left(2 \mathrm{H}, \mathrm{m}, \mathrm{C}(\mathrm{O}) \mathrm{CHCH}_{2}\right), 1.80$ $-1.55\left(7 \mathrm{H}, \mathrm{C}(\mathrm{O}) \mathrm{CHCH}_{2} \mathrm{CH}_{2}, \mathrm{CHCH}_{2} \mathrm{CH}_{3}, \mathrm{CHCHCH}_{3}\right), 1.18\left(3 \mathrm{H}, \mathrm{t}, \mathrm{J}=7.4 \mathrm{~Hz}, \mathrm{CH}_{2} \mathrm{CH}_{3}\right)$. 


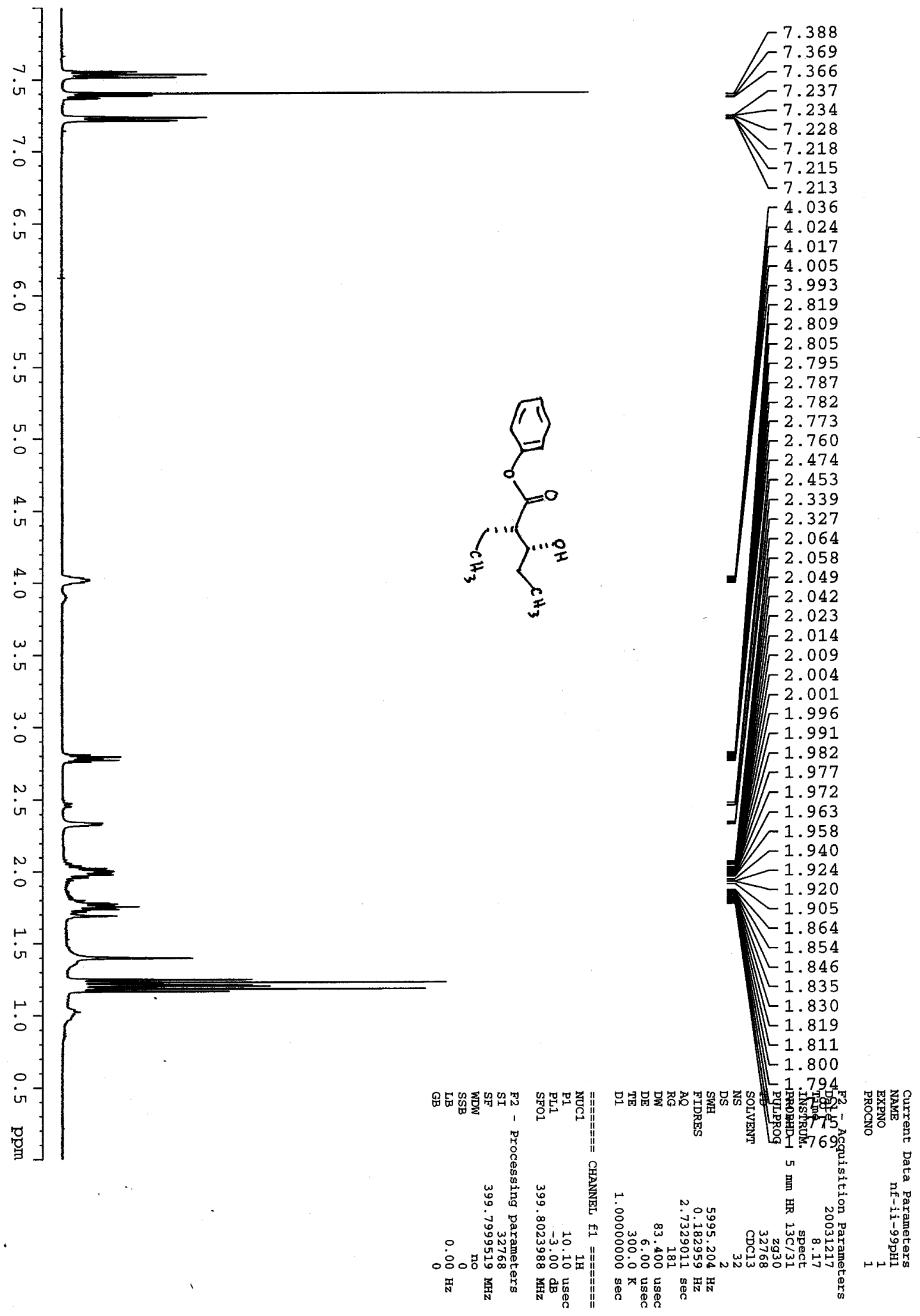




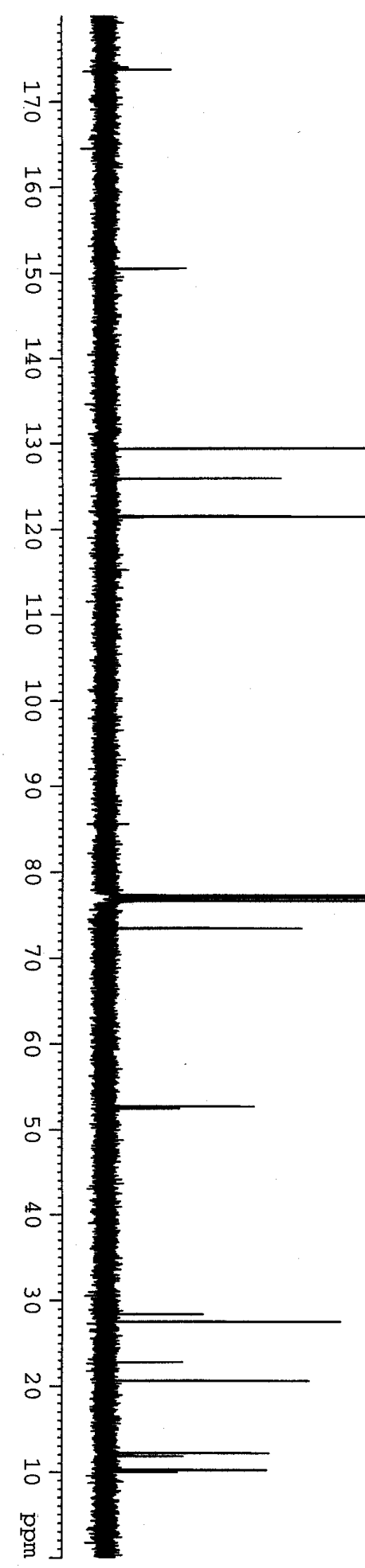

173.708

150.496
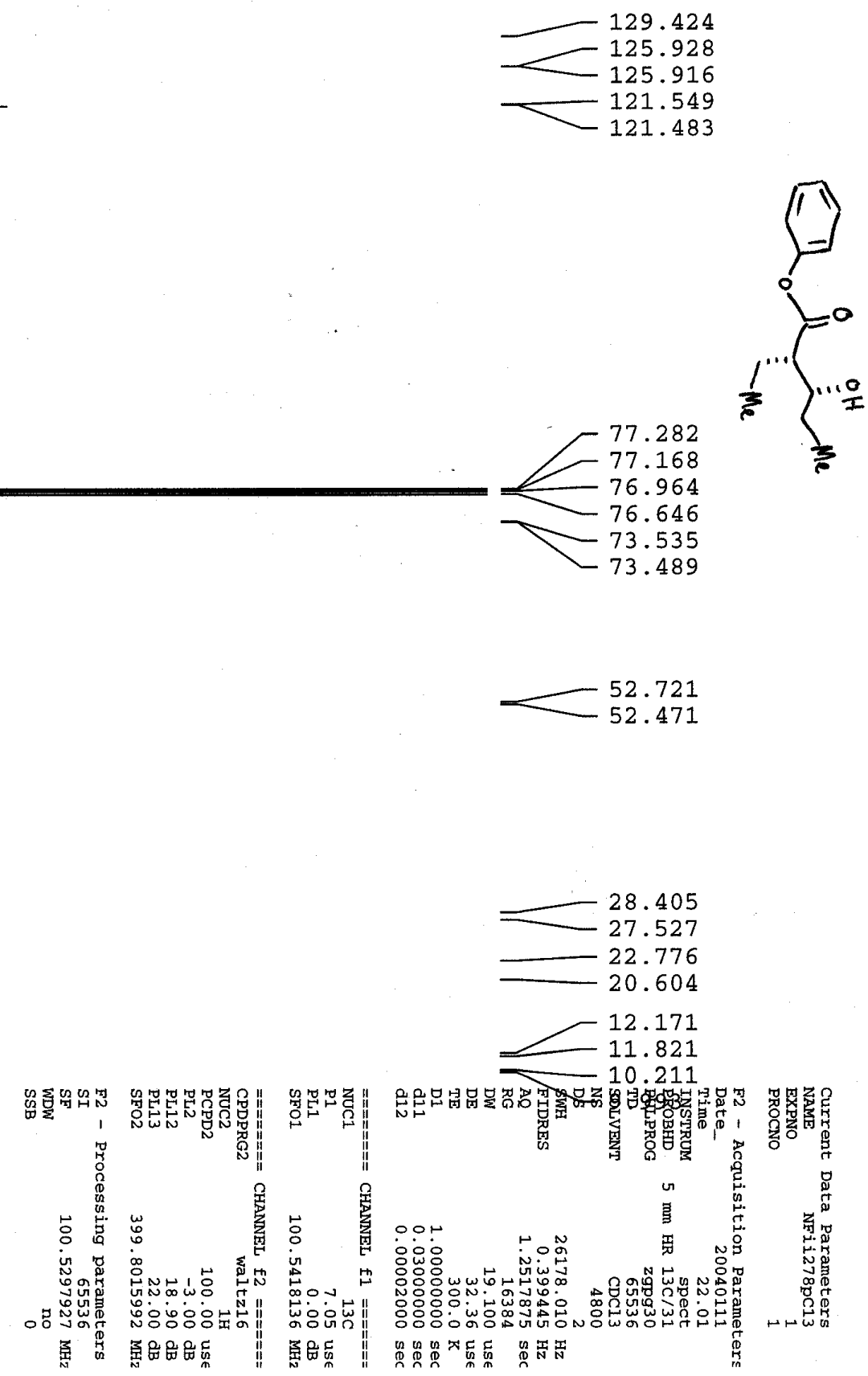


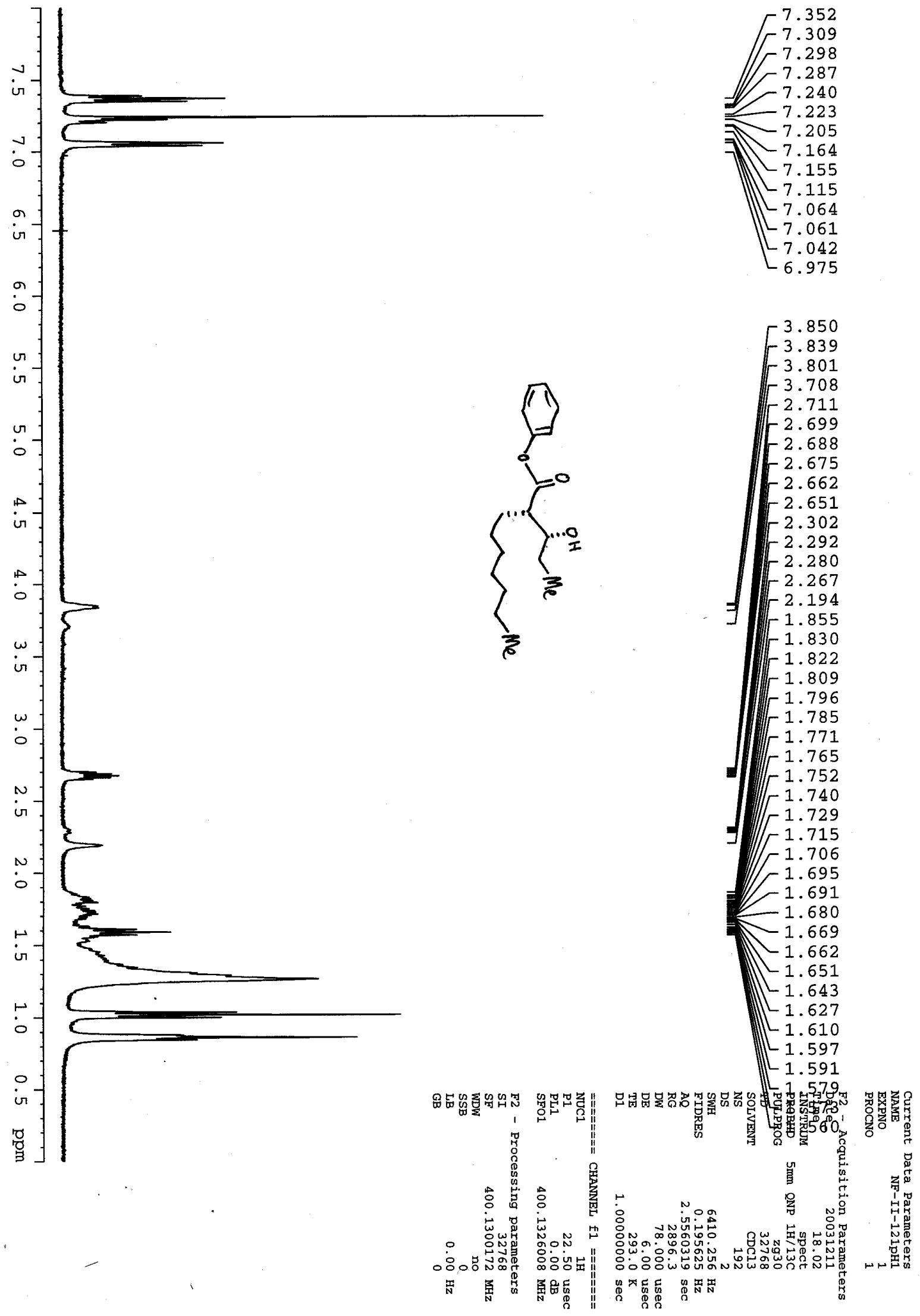




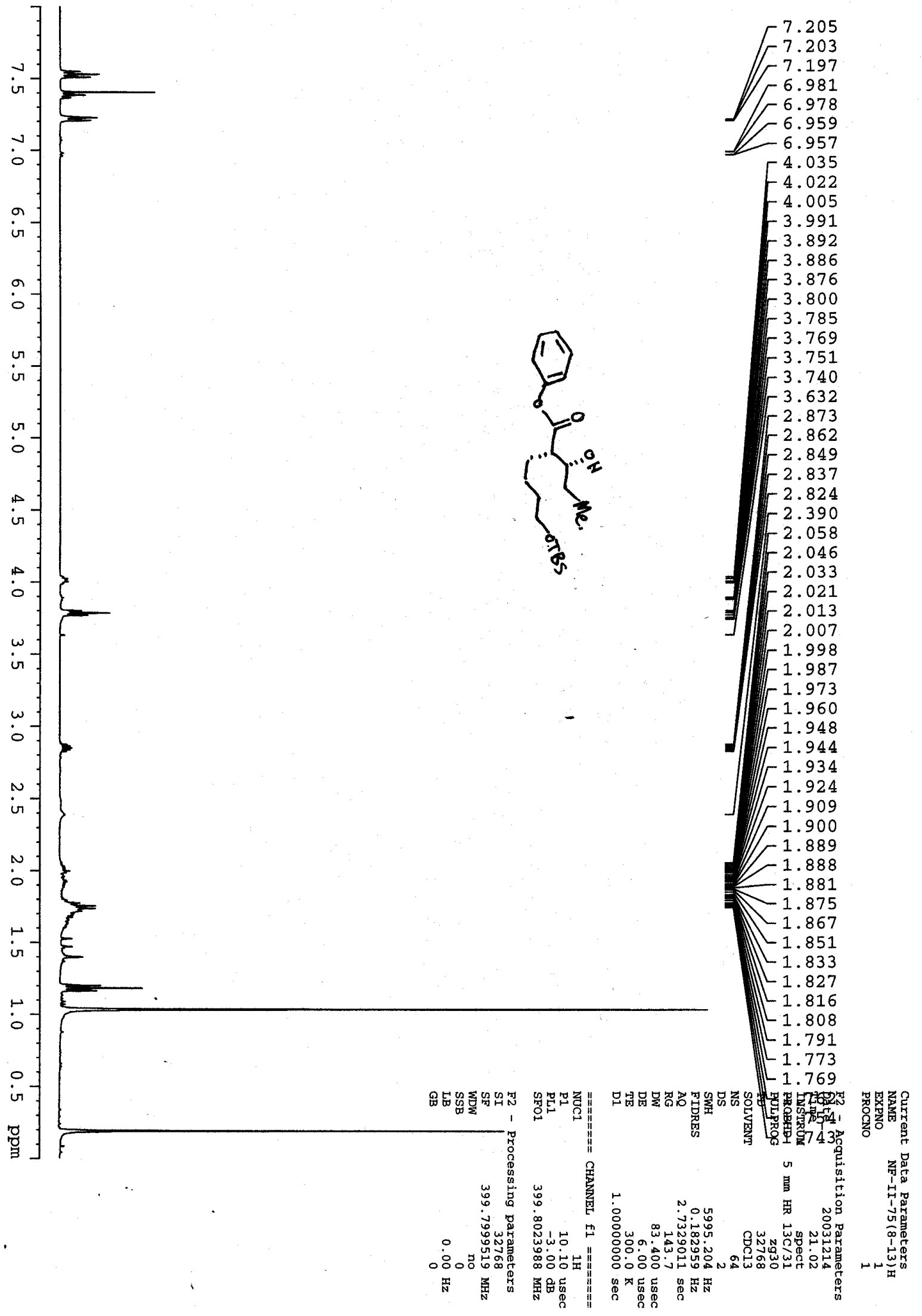




$$
E 1
$$



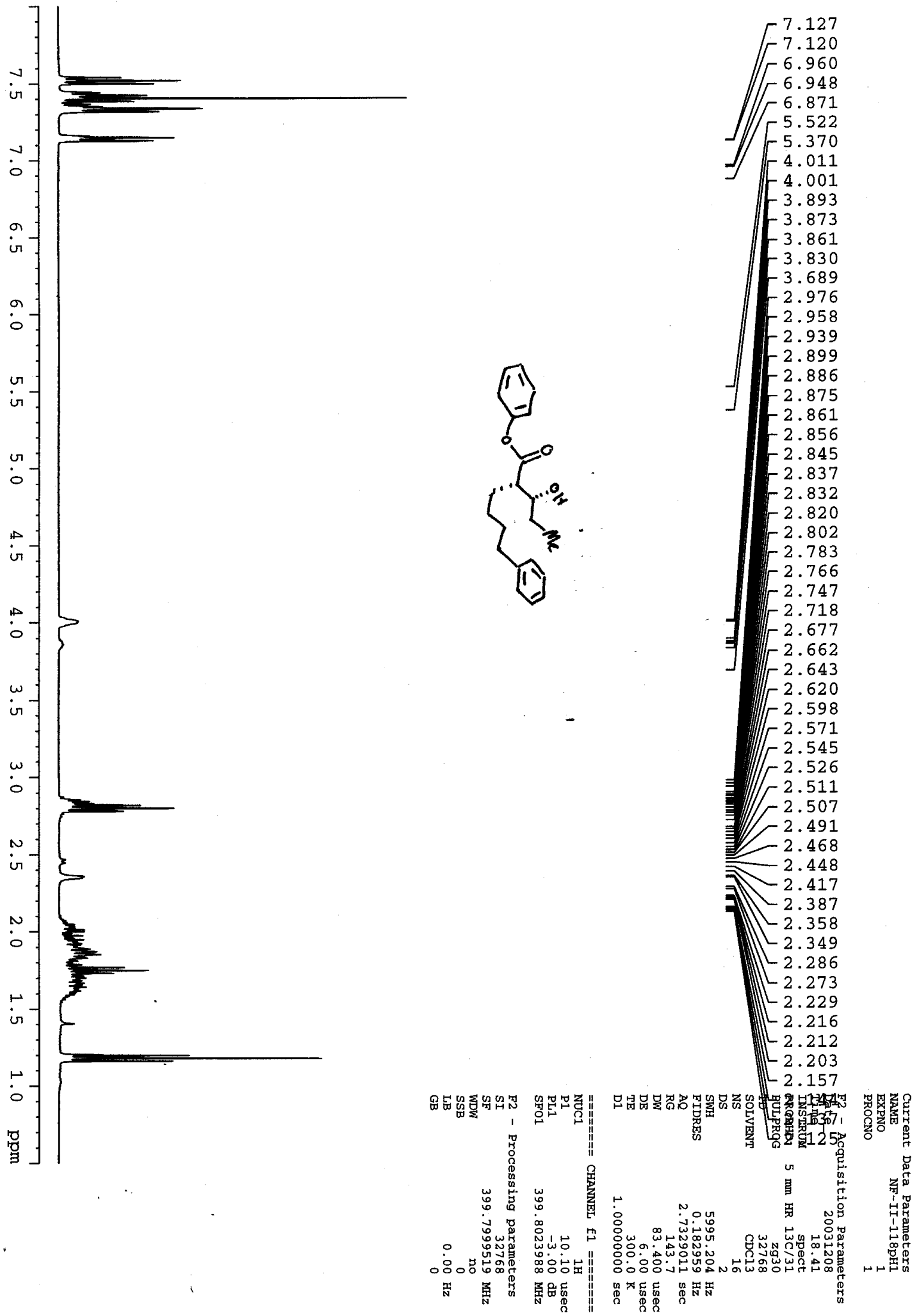


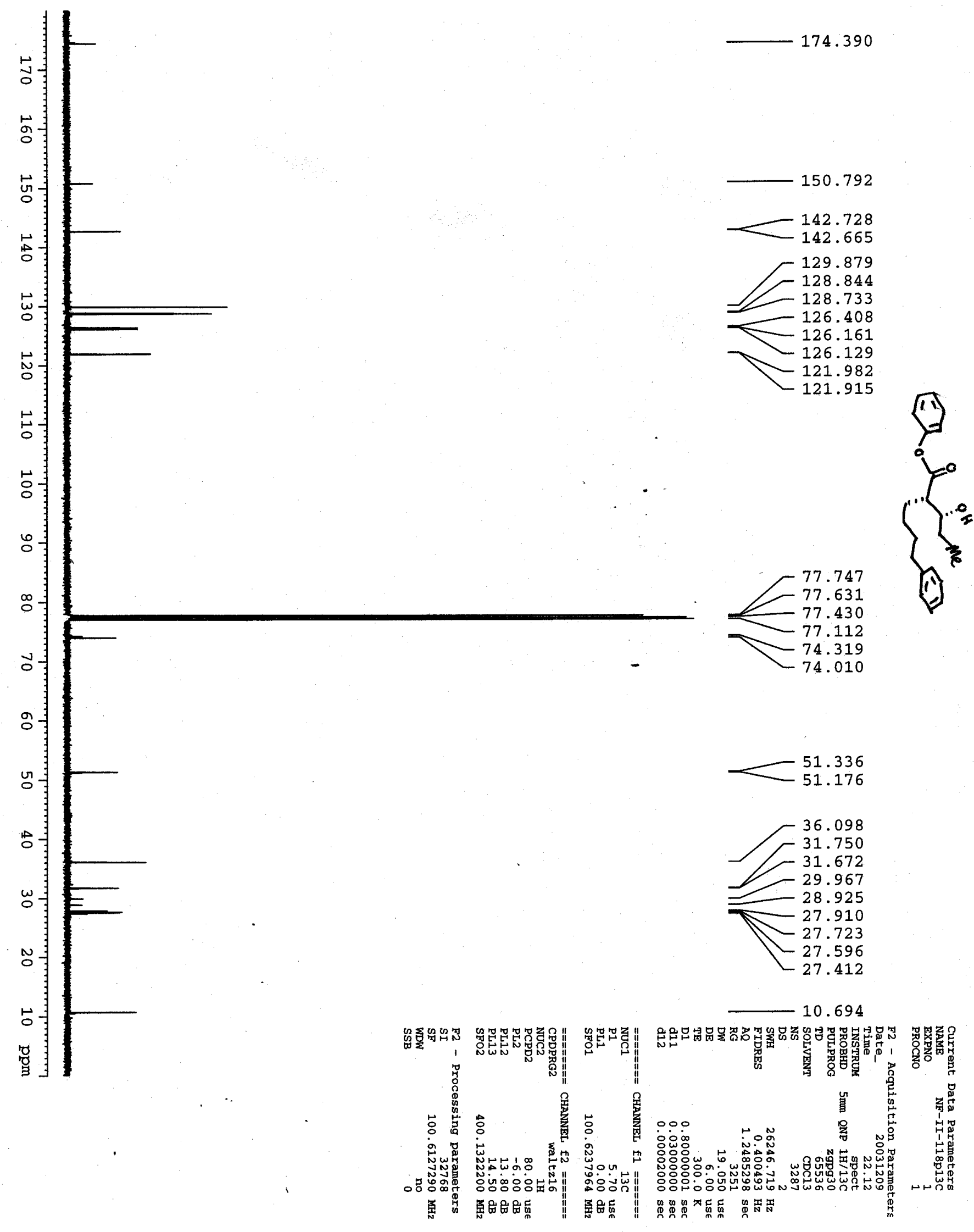

